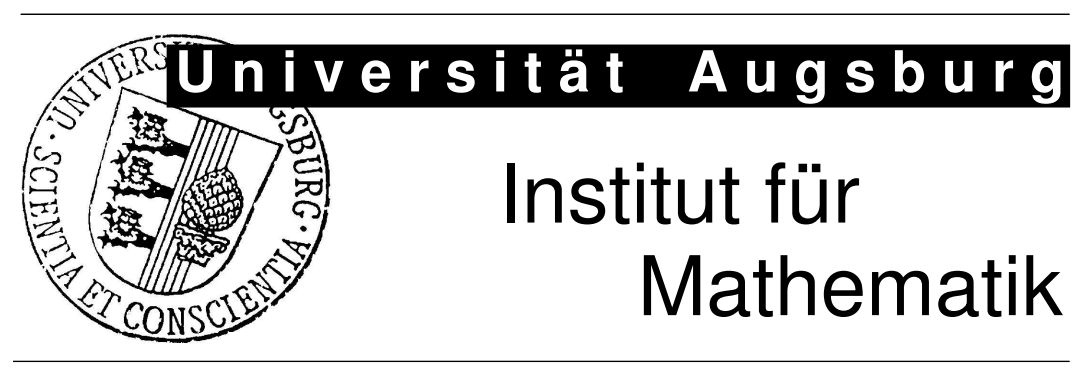

Oleg Boyarkin, Ronald H. W. Hoppe

Optimal Control of Inductive Heating of Ferromagnetic Materials

Preprint Nr. 10/2013 - 06. Mai 2013

Institut für Mathematik, Universitätsstraße, D-86135 Augsburg

http: //www .math. uni-augsburg.de/ 


\section{Impressum:}

\section{Herausgeber:}

Institut für Mathematik

Universität Augsburg

86135 Augsburg

http://www . math. uni-augsburg.de/de/forschung/preprints.html

\section{ViSdP:}

Ronald H. W. Hoppe

Institut für Mathematik

Universität Augsburg

86135 Augsburg

Preprint: Sämtliche Rechte verbleiben den Autoren (C) 2013 


\title{
OPTIMAL CONTROL OF INDUCTIVE HEATING OF FERROMAGNETIC MATERIALS
}

\author{
O. BOYARKIN* ${ }^{*}$ AND R. H. W. HOPPE*†ई
}

\begin{abstract}
Inductive heating is a technological process where a steel workpiece is surrounded by an electromagnetic coil to which currents at various frequencies and time-varying amplitudes are applied. The amplitudes are considered as the controls and the objective is to heat the workpiece up to a desired temperature profile at the final time of the heating process. The workpiece is then quenched which due to a phase transition in the crystallographic structure of the steel leads to a hardening of the surface of the workpiece. For the inductive heating process, the state equations represent a coupled system of nonlinear partial differential equations consisting of the eddy currents equations in the coil, the workpiece, and the surrounding air, and a heat equation in the workpiece. The nonlinearity stems from the temperature dependent nonlinear material laws for steel both with regard to its electromagnetic and thermal behavior. Following the principle 'Discretize first, then optimize', we consider a semi-discretization in time by the implicit Euler scheme which leads to a discrete-time optimal control problem. We prove the existence of a minimizer for the discrete-time optimal control problem and derive the first order necessary optimality conditions.
\end{abstract}

Keywords: optimal control, inductive heating, eddy currents equations, heat equation

\section{AMS subject classification: 49M05, 65K10, 65M60, 78M10}

1. Introduction. Induction heating of mechanical tools made from steel is a modern processing technology used for surface hardening. The boundary layer of the tool is rapidly heated up via electromagnetic waves to temperatures above the Curie temperature where steel undergoes a phase transition. This is followed by quenching which leads to a change in the crystallographic structure from austenite to martensite and hence to an increased hardness of the tool in a region close to the surface. Most contributions in the literature are concerned with numerical simulations of the inductive heating process (cf., e.g., $[2,5,6,9,13,14,17,18,19,22])$.
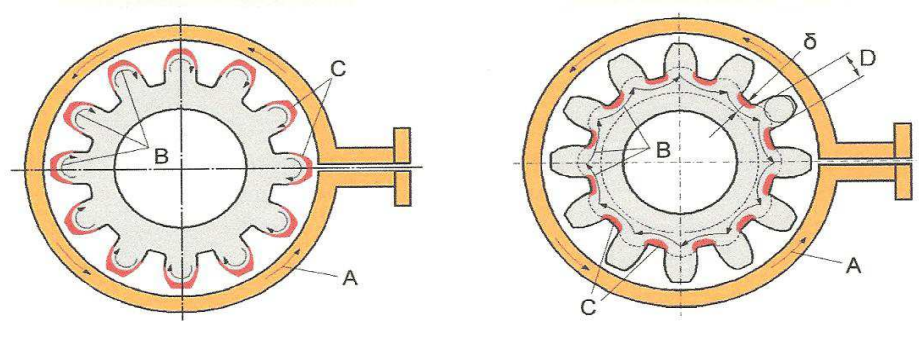

FIG. 1.1. Induction heating at high frequency (left) and medium frequency (right). At high frequency only the tips of the teeth are heated up, whereas at medium frequency most heating occurs at the interstitial space between the teeth (cf. region $C$ in the left and right figure)

*Inst. of Math., Univ. of Augsburg, D-86159 Augsburg, Germany

E-mail: thomas.fraunholz@math.uni-augsburg.de, hoppe@math.uni-augsburg.de

${ }^{\dagger}$ Dept. of Math., Univ. of Houston, Houston, TX 77204-3008, U.S.A.

E-mail: rohop@math.uh.edu

${ }^{\ddagger}$ Supported by the BMBF within the Collaborative Research Project 'MeFreSim'.

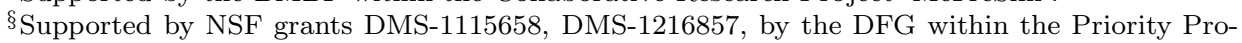
gram SPP 1506, by the BMBF within the Collaborative Research Projects 'FROPT' and 'MeFreSim', and by the ESF within the Research Networking Programme 'OPTPDE'. 
The apparatus consists of a copper made coil around the tool as shown in Figure 1.1. Applying a voltage at the coil, an electromagnetic field is generated which penetrates the tool. Electromagnetic energy is converted to Joule heat which primarily happens in a region close to the surface due to the skin effect. For tools with a somewhat complicated surface geometry such as a gear, the main goal is to guarantee a uniform temperature distribution and thus a uniform hardness in the boundary layer. The penetration depth essentially depends on the distance between the coil and the gear and on the chosen frequency. Using just one frequency, a uniform temperature distribution cannot be achieved: a high frequency $(100 \mathrm{kHz})$ only leads to a heating of the tips of the teeth, whereas the heating at a medium frequency $(20 \mathrm{kHz})$ is restricted to the base of the teeth (cf. Figure 1.1). Therefore, the current technology is based on the simultaneous feed-in of currents of possibly time-varying amplitudes [23, 26]. It is the purpose of this paper to study the optimal control of the amplitudes such that at the final time of the inductive heating a desired temperature profile is achieved in the workpiece. We note that from an engineering point of view the optimal control of inductive heating processes has been addressed in [24], whereas a more analytical oriented treatment can be found in [8], but not for a ferromagnetic material with its inherent nonlinear material behavior. Here, we consider inductive heating processes where the computational domain $D$ is a bounded domain in $\mathbb{R}^{3}$ containing the domain $\Omega_{1}$ occupied by the coil, the domain $\Omega_{2}$ occupied by the steel workpiece, and a domain $\Omega_{3}:=D \backslash\left(\bar{\Omega}_{1} \cup \bar{\Omega}_{2}\right)$ which contains the surrounding air. The state equations represent a system of partial differential equations consisting of the eddy current equations in $D$ coupled with a heat equation in $\Omega_{2}$.

The paper is organized as follows: Section 2 is devoted to the derivation of the state equations. In particular, in subsection 2.1 we present the eddy currents equations based on a generalized Fröhlich's model for the nonlinear relationship between the magnetic induction and the magnetic field in ferromagnetic materials, whereas subsection 2.2 deals with the heat equation. Based on a semi-discretization in time of the state equations and a linearization of the nonlinear material behavior, the discretetime optimal control problem is stated in section 3. We prove the existence and uniqueness of a weak solution of the discrete-time state equations, whereas the existence of an optimal solution of the discrete-time control problem is shown in section 4 . In section 5 we are concerned with the derivation of the associated optimality system in terms of the discrete-time adjoint system and the discrete-time gradient equation. Finally, section 6 briefly discusses the spatial discretization by finite elements with respect to a geometrically conforming simplicial triangulation of the computational domain and provides a documentation of numerical results.

1.1. Preliminaries. We use standard notations from Lebesgue and Sobolev space theory [28]. In particular, given a bounded Lipschitz domain $\Omega \subset \mathbb{R}^{3}$ with boundary $\Gamma:=\partial \Omega$, we refer to $L^{p}(\Omega), 1 \leq p \leq \infty$, as the Banach spaces of $p$-th power integrable functions $(p<\infty)$ and essentially bounded functions $(p=\infty)$ on $\Omega$ with norm $\|\cdot\|_{0, p, \Omega}$, respectively. In case $p=2$, the space $L^{2}(\Omega)$ is a Hilbert space whose inner product and norm will be referred to as $(\cdot, \cdot)_{0, \Omega}$ and $\|\cdot\|_{0, \Omega}$. For $s \in \mathbb{R}_{+}$, we denote by $W^{s, p}(\Omega)$ the Sobolev spaces with norms $\|\cdot\|_{s, p, \Omega}$ and associated seminorms $|\cdot|_{s, p, \Omega}$. We refer to $W_{0}^{s, p}(\Omega)$ as the closure of $C_{0}^{\infty}(\Omega)$ in $W^{s, p}(\Omega)$. For $s<0$, we denote by $W^{s, p}(\Omega)$ the dual space of $W_{0}^{-s, q}(\Omega), p^{-1}+q^{-1}=1$. In case $p=2$, the spaces $W^{s, 2}(\Omega)$ are Hilbert spaces. We will write $H^{s}(\Omega)$ instead of $W^{s, 2}(\Omega)$ and refer to $(\cdot, \cdot)_{s, \Omega}$ and $\|\cdot\|_{s, \Omega}$ as the inner products and associated norms. We denote by $C(\Omega)$ the Banach space of continuous functions and refer to $\mathcal{M}(\Omega)$ as its dual 
space of regular Borel measures. In case of vector-valued functions, the above function spaces can be defined analogously and will be denoted in boldface, e.g., $\mathbf{L}^{2}(\Omega)$ stands for the Hilbert space of vector-valued functions on $\Omega$ with square-integrable components. In particular, $\mathbf{H}(\mathbf{c u r l}, \Omega)$ is the Hilbert space of functions $\mathbf{q} \in \mathbf{L}^{2}(\Omega)$ with curl $\mathbf{q} \in \mathbf{L}^{2}(\Omega)$, equipped with the graph norm.

Moreover, for $T>0$ we consider the space-time domain $Q:=\Omega \times(0, T)$. Given a Banach space $X$, we denote by $L^{p}((0, T), X), 1 \leq p \leq \infty$, and $C([0, T], X)$ the Banach spaces of functions $v:[0, T] \rightarrow X$. The spaces $W^{s, p}((0, T), X)$ and $H^{s}((0, T), X), s \in$ $\mathbb{R}_{+}$, are defined analogously.

2. The Inductive Heating Process. Let $D:=B_{r}(0) \subset \mathbb{R}^{3}$ be a ball centered at the origin with radius $r>0$ containing a torus $\Omega_{1}:=B_{r_{1}}(a) \times(0,2 \pi), a:=$ $\left(r_{2}, 0,0\right)^{T}, r_{1}+r_{2}<r$, representing the coil, and a cylindrical domain $\Omega_{2}:=S \times$ $(-h / 2,+h / 2), h>0$, with cross section $S$ centered at the origin such that $\operatorname{diam}(S)<$ $r_{2}$, representing the steel workpiece. The domain $\Omega_{3}:=D \backslash\left(\bar{\Omega}_{1} \cup \bar{\Omega}_{2}\right)$ is supposed to be filled with air. We further refer to $\partial D, \Gamma_{1}:=\partial \Omega_{1}, \Gamma_{2}:=\partial \Omega_{2}$, and $\Gamma_{3}:=\partial D \cup \Gamma_{1} \cup \Gamma_{2}$ as the boundary of $D$, the boundary of $\Omega_{1}, \Omega_{2}$, and of $\Omega_{3}$, respectively. We note that the boundaries $\partial D$ and $\Gamma_{1}$ are of class $C^{\infty}$, whereas we assume $\Gamma_{2}$ to be of class $C^{2}$. Finally, for $T>0$ we set $Q:=D \times(0, T), Q_{i}:=\Omega_{i} \times(0, T), 1 \leq i \leq 3$, and $\Sigma:=\partial D \times(0, T), \Sigma_{i}:=\Gamma_{i} \times(0, T), 1 \leq i \leq 3$.

The inductive heating processes can be described by Maxwell's equations

$$
\operatorname{curl} \mathbf{H}=\mathbf{J}+\frac{\partial \mathbf{D}}{\partial t}, \quad \operatorname{curl} \mathbf{E}=-\frac{\partial \mathbf{B}}{\partial t}, \quad \operatorname{div} \mathbf{B}=0 \quad \text { in } Q,
$$

coupled with a heat equation

$$
\rho c(\theta) \frac{\partial \theta}{\partial t}-\operatorname{div}(\kappa(\theta) \operatorname{grad} \theta)=f \quad \text { in } Q_{2} .
$$

Here, $\mathbf{E}$ and $\mathbf{H}$ stand for the electric and the magnetic field, $\mathbf{B}, \mathbf{D}$, and $\mathbf{J}$ refer to the magnetic flux density, the dielectric displacement, and the current density. Moreover, $\theta$ denotes the temperature, $\rho$ and $c(\theta), \kappa(\theta)$ are the mass density and the temperature dependent heat capacity and thermal conductivity, respectively. Finally, the source term $f$ in $(2.2)$ stands for the Joule heat.

2.1. The Eddy Currents Equations. In the non-magnetic domains $\Omega_{1}$ (conductor, made from copper) and $\Omega_{3}$ (air), the vector fields $\mathbf{B}_{\nu}, \mathbf{H}_{\nu}, \mathbf{D}_{\nu}, \mathbf{E}_{\nu}$, and $\mathbf{J}_{\nu}$ are related by the linear material laws

$$
\mathbf{B}_{\nu}=\mu_{\nu} \mathbf{H}_{\nu}, \quad \mathbf{D}_{\nu}=\varepsilon_{\nu} \mathbf{E}_{\nu}, \quad \mathbf{J}_{\nu}=\sigma_{\nu} \mathbf{E}_{\nu}, \quad \nu \in\{1,3\}
$$

Here, $\varepsilon_{\nu}$ and $\mu_{\nu}$ are positive constants denoting the electric permittivity and the magnetic permeability, whereas $\sigma_{\nu}$ stand for the electric conductivities which are positive constants in $\Omega_{\nu}$ with $\sigma_{3} \ll \sigma_{1}$. The positive constants $\xi_{\nu}:=\mu_{\nu}^{-1}$ and $\rho_{\nu}:=$ $\sigma_{\nu}^{-1}, \nu \in\{1,3\}$, are referred to as the magnetic susceptibilities and electric resistivities, respectively.

However, in $\Omega_{2}$ (steel workpiece) there is a temperature dependent nonlinear $\mathbf{B}-\mathbf{H}$ relationship. Here, we use the generalized Fröhlich's model [27]

$$
\mathbf{B}_{2}=\frac{\mathbf{H}_{2}}{\alpha(\theta)+\beta(\theta)\left|\mathbf{H}_{2}\right|},
$$



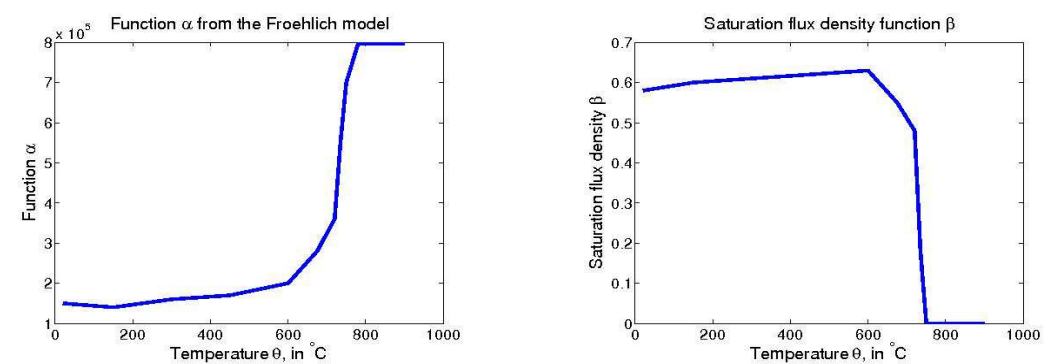

FIG. 2.1. Temperature dependence of the function $\alpha$ and the saturation flux density $\beta$ in the generalized Fröhlich's model.

where the function $\alpha(\theta)$ is a monotonically increasing function in $\theta$ which approaches a positive constant $\xi_{2}=\mu_{2}^{-1}$ after $\theta$ has passed the Curie temperature $\theta_{C}$ (cf. Figure 2.1 (left)).

Moreover, the saturation flux density $\beta(\theta)$ is a continuous function in $\theta$ which first slightly increases and then monotonically decreases for $\theta<\theta_{C}$ and $\beta(\theta)=0$ for $\theta \geq \theta_{C}$ with a jump discontinuity of its first derivative at $\theta_{C}$ (cf. Figure 2.1 (right)). We use mollified versions of $\alpha(\theta)$ and $\beta(\theta)$ in terms of standard Friedrichs' mollifiers around $\theta_{C}$. Solving for $\mathbf{H}_{2}$, we obtain

$$
\mathbf{H}_{2}=\xi_{2}\left(\theta,\left|\mathbf{B}_{2}\right|\right) \mathbf{B}_{2}, \quad \xi_{2}\left(\theta,\left|\mathbf{B}_{2}\right|\right)=\frac{\alpha(\theta)}{1-\beta(\theta)\left|\mathbf{B}_{2}\right|} .
$$

We assume that there exist constants $0<c<C$ such that

$$
c \leq \xi_{2}\left(\theta,\left|\mathbf{B}_{2}\right|\right) \leq C, \quad\left|\xi_{2, \theta}\left(\theta,\left|\mathbf{B}_{2}\right|\right)\right| \leq C, \quad\left|\xi_{2,\left|\mathbf{B}_{2}\right|}\left(\theta,\left|\mathbf{B}_{2}\right|\right)\right| \leq C,
$$

over the whole range of the temperature $\theta$ and the magnetic induction $\mathbf{B}_{2}$ during the inductive heating process, where $\xi_{2, \theta}$ and $\xi_{2,\left|\mathbf{B}_{2}\right|}$ stand for the derivatives of $\xi_{2}$ with respect to $\theta$ and $\left|\mathbf{B}_{2}\right|$.

REMARK 2.1. An alternative to the generalized Fröhlich's model (2.5) is a model suggested by Bristiel [3] which reads

$$
\mathbf{B}_{2}=\frac{2 J_{S}}{\pi}\left(1-\exp \left(\left(\theta-\theta_{C}\right) / C\right) \arctan \left(\frac{(a-1) \pi \mu_{2} \mathbf{H}_{2}}{2 J_{S}}\right)+\mu_{2} \mathbf{H}_{2},\right.
$$

where $a, C$, and $J_{S}$ are suitably chosen parameters.

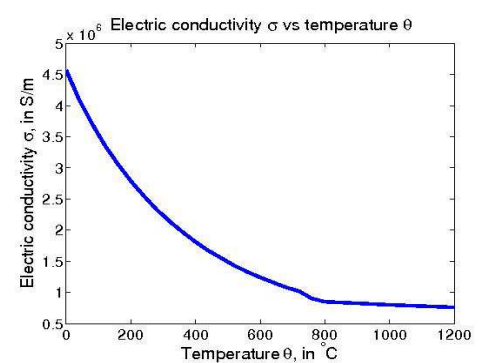

FIG. 2.2. Temperature dependence of the electric conductivity $\sigma$ 
Moreover, in $\Omega_{2}$ the conductivity $\sigma_{2}$ depends on the temperature $\theta$ and represents a smooth monotonically decreasing positive function $\sigma_{2}(\theta)$ decreasing from approximately $4.5 \cdot 10^{6} \mathrm{~S} / \mathrm{m}$ at $\theta=0^{\circ} C$ to approximately $0.5 \cdot 10^{6} \mathrm{~S} / \mathrm{m}$ at $1200^{\circ} C$ (cf. Figure $2.2)$. Hence, we may assume

$$
c \leq \sigma_{2}(\theta) \leq C
$$

over the whole range of the temperature $\theta$ during the inductive heating process.

We use the low frequency approximation of Maxwell's equations, where the dielectric field $\mathbf{D}$ is neglected. We introduce a magnetic vector potential $\mathbf{A}$ and a time integrated electric scalar potential $\varphi$ according to

$$
\mathbf{B}=\operatorname{curl} \mathbf{A}, \quad \mathbf{E}=-\frac{\partial \mathbf{A}}{\partial t}-\operatorname{grad} \frac{\partial \varphi}{\partial t} .
$$

Setting $\mathbf{A}_{\nu}:=\left.\mathbf{A}\right|_{\Omega_{\nu}}$, this results in the eddy currents equations

$$
\sigma_{\nu} \frac{\partial \mathbf{A}_{\nu}}{\partial t}+\operatorname{curl}\left(\xi_{\nu} \operatorname{curl} \mathbf{A}_{1}\right)+\sigma_{\nu} \operatorname{grad} \frac{\partial \varphi}{\partial t}=\mathbf{0} \in Q_{1}
$$

For a theoretical justification of the eddy currents equations we refer to [1]. The time integrated scalar electric potential is of the form

$$
\varphi(x, t)=\left\{\begin{array}{rll}
h(x) & \int_{0}^{t}\left(g_{1}(\tau)+g_{2}(\tau)\right) d \tau & \text { in } \bar{Q}_{1} \\
& 0 & \text { in } \bar{Q} \backslash \bar{Q}_{1}
\end{array} .\right.
$$

Here, $h \in C_{0}^{\infty}\left(\Omega_{1}\right)$ and $g_{i}(\tau)=u_{i}(\tau) U_{i} \cos \left(\omega_{i} \tau\right), 1 \leq i \leq 2$, where $U_{1}, U_{2}$ are the maximum voltage outputs corresponding to the high frequency $\omega_{1}$ and the medium frequency $\omega_{2}$ and the functions $u_{1}(t), u_{2}(t)$ stand for the fractions of the maximum power outputs that are used to control the induction heating process.

For $\sigma_{3}=0$ we note that $(2.10 \mathrm{a})$ is not well defined in $\Omega_{3}$ due to the nontrivial kernel of the curl operator. We impose a Coulomb gauge

$$
\operatorname{div} \mathbf{A}_{\nu}=0 \text { in } Q_{\nu}, 1 \leq \nu \leq 3,
$$

for all equations (2.10). Using (2.11) and (2.12) in (2.10) and setting

$$
\mathbf{u}(t)=\left(u_{1}(t), u_{2}(t)\right)^{T}, \quad \boldsymbol{\psi}(t)=\left(\psi_{1}(t), \psi_{2}(t)\right)^{T}, \psi_{i}(t):=U_{i} \cos \left(\omega_{i} \tau\right), 1 \leq i \leq 2,
$$

and $\mathbf{j}:=-\operatorname{grad} h$, we obtain

$$
\sigma_{\nu} \frac{\partial \mathbf{A}_{\nu}}{\partial t}+\operatorname{curl}\left(\xi_{\nu} \operatorname{curl} \mathbf{A}_{1}\right)-\operatorname{grad} \operatorname{div} \mathbf{A}_{\nu}=\delta_{\nu 1} \mathbf{u}(t) \cdot \boldsymbol{\psi}(t) \mathbf{j} \quad \text { in } Q_{\nu}
$$

The eddy currents equations (2.13a) have to be complemented by a boundary condition on $\Sigma$

$$
\mathbf{A}_{3}=\mathbf{0} \text { on } \Sigma,
$$

transmission conditions on $\Sigma_{i}, 1 \leq i \leq 2$,

$$
\mathbf{n}_{\Gamma_{i}} \wedge\left(\xi_{i} \text { curl }\left.\mathbf{A}\right|_{\bar{\Omega}_{i} \cap \Gamma_{i}} \wedge \mathbf{n}_{\Gamma_{i}}\right) \begin{array}{r}
\left.\mathbf{A}\right|_{\bar{\Omega}_{i} \cap \Gamma_{i}} \wedge \mathbf{n}_{\Gamma_{i}}=-\left.\mathbf{A}\right|_{\bar{\Omega}_{3} \cap \Gamma_{3}} \wedge \mathbf{n}_{\Gamma_{i}} \\
\mathbf{n}_{\Gamma_{3}} \wedge\left(\left.\xi_{3} \operatorname{curl} \mathbf{A}\right|_{\bar{\Omega}_{3} \cap \Gamma_{3}} \wedge \mathbf{n}_{\Gamma_{3}}\right) \\
\left.\mathbf{n}_{\Gamma_{i}} \cdot \mathbf{A}\right|_{\bar{\Omega}_{i} \cap \Gamma_{i}}=-\left.\mathbf{n}_{\Gamma_{3}} \cdot \mathbf{A}\right|_{\bar{\Omega}_{i} \cap \Gamma_{3}}
\end{array}
$$


and an initial condition at $t=0$

$$
\mathbf{A}(\cdot, 0)=\mathbf{0} \text { in } D
$$

REMARK 2.2. Instead of imposing the Coulomb gauge throughout the entire computational domain, it suffices to enforce it weakly only in the subdomain $\Omega_{3}$, i.e., we are looking for a pair $\left(\mathbf{A}_{3}, \varphi_{3}\right) \in \mathbf{H}_{0, \partial D}\left(\mathbf{c u r l}, \Omega_{3}\right) \times H_{0}^{1}\left(\Omega_{3}\right)$ such that for all $\mathbf{q} \in \mathbf{H}_{0, \partial D}\left(\mathbf{c u r l}, \Omega_{3}\right)$ and all $\psi \in H_{0}^{1}\left(\Omega_{3}\right)$ it holds

$$
\begin{aligned}
& \left(\xi_{3} \operatorname{curl} \mathbf{A}_{3}, \operatorname{curl} \mathbf{q}\right)_{0, \Omega_{3}}+\left(\mathbf{q}, \operatorname{grad} \varphi_{3}\right)_{0, \Omega_{3}}=\ell_{3}(\mathbf{q}), \\
& \left(\mathbf{A}_{3}, \operatorname{grad} \psi\right)_{0, \Omega_{3}}=0 \text {, }
\end{aligned}
$$

where the right-hand side $\ell_{3}(\mathbf{q})$ in $(2.14 \mathrm{a})$ is given in terms of the second transmission condition in $(2.13 \mathrm{c})$ and thus provides the coupling with the eddy current equations in $\Omega_{1}$ and $\Omega_{2}$.

2.2. The Heat Equation. The specific heat capacity $q(\theta):=\rho c(\theta)$ in $(2.2)$ is a monotonically increasing function for $\theta<\theta_{C}$ and $\theta>\theta_{C}$, but exhibits a jump discontinuity approximately at the Curie temperature $\theta_{C}$ (cf. Figure 2.3 (left)). We use a mollified heat capacity in terms of a standard Friedrichs-type mollifier such that $q(\theta)=\rho c(\theta)$ becomes a smooth function in $\theta$ satisfying

$$
c \leq q(\theta) \leq C
$$

over the range of the temperature during the inductive heating process. The thermal conductivity $\kappa$ depends on $\theta$ as well and represents a continuous function that is monotonically decreasing from approximately $43 \mathrm{~W} /(\mathrm{mK})$ at room temperature to $27 \mathrm{~W} /(\mathrm{mK})$ at the Curie temperature and then moderately increasing to $30 \mathrm{~W} /(\mathrm{mK})$ at $1200^{\circ} \mathrm{C}$ (cf. Figure 2.3 (right)). We also smooth out the discontinuity of the derivative of $\kappa(\theta)$ at $\theta_{C}$ such that $\kappa(\theta)$ becomes a smooth function, and we assume

$$
c \leq \kappa(\theta) \leq C
$$
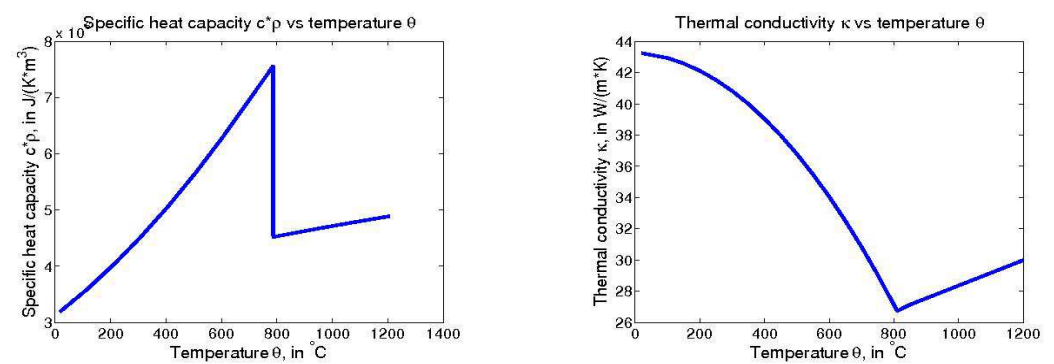

FIG. 2.3. Temperature dependence of the specific heat capacity $q=\rho c$ (left) and of the thermal conductivity $\kappa$ (right)

Moreover, the source $f$ in (2.2) (Joule heat) is given by

$$
f\left(\mathbf{A}_{2}\right):=\sigma_{2}\left|\frac{\partial \mathbf{A}_{2}}{\partial t}\right|^{2} .
$$


The heat equation takes the form:

$$
q(\theta) \frac{\partial \theta}{\partial t}-\operatorname{div}(\kappa(\theta) \operatorname{grad} \theta)=f\left(\mathbf{A}_{2}\right) \quad \text { in } Q_{2}
$$

It is complemented by the Robin-type boundary condition

$$
\mathbf{n}_{\Gamma_{2}} \cdot \kappa(\theta) \operatorname{grad} \theta+h_{s}\left(\theta-\theta_{a}\right)^{\gamma}+\sigma_{S B} \varepsilon\left(|\theta|^{3} \theta-\theta_{a}^{4}\right)=0 \quad \text { on } \Sigma_{2},
$$

and by the initial condition

$$
\theta(\cdot, 0)=\theta_{0} \quad \text { in } \Omega_{2}
$$

We note that $(2.18 \mathrm{~b})$ takes into account heat losses by convective and radiation heat transfer. Here, $h_{s}$ is the convection surface heat transfer coefficient, $\sigma_{S B}$ is the StefanBoltzmann constant, $\varepsilon$ denotes the emissivity of the surface, and $\theta_{a}$ stands for the ambient temperature. For a steel/open air surface, we typically have $h_{s} \approx 1.54$, $\gamma \approx 1.33$ and $\varepsilon$ is varying between 0.03 and 0.7 depending on the properties of the surface [7]. The ambient temperature $\theta_{a}$ and the initial temperature $\theta_{0}$ are supposed to be positive constants.

\section{The Optimal Control Problem.}

3.1. The Discrete-Time Optimal Control Problem. We consider a partition $\mathcal{T}_{T}:=\left\{0=: t_{0}<t_{1}<\cdots<t_{M}:=T\right\}$ of the time interval $[0, T]$ into subintervals $\left[t_{m-1}, t_{m}\right], 1 \leq m \leq M$, of length $\Delta t:=T / M, M \in \mathbb{N}$. The controls are vectors $\mathbf{u}^{m} \in \mathbb{R}^{2}$ at the time instants $t_{m}, 1 \leq m \leq M$, of the partition $\mathcal{T}_{T}$. We denote by $\mathbf{A}^{m}$ and $\theta^{m}$ approximations of $\mathbf{A}$ and $\theta$ at $t=t_{m}, 1 \leq m \leq M$. We linearize the state equations by 'freezing' the coefficients $\sigma, \xi$ in the eddy currents equations (2.13) and the coefficients $q, \kappa$ in the heat equation (2.18) and by linearizing the convective and radiative heat transfer in $(2.18 \mathrm{~b})$.

To this end, we define $\left|\tilde{\mathbf{B}}_{2}^{m-1}\right|$ and $\tilde{\theta}^{m-1}$ as the average of $\left|\mathbf{B}_{2}^{m-1}\right|$ and $\theta^{m-1}$ over $\Omega_{2}$. Moreover, $\tilde{\theta}_{s}^{m-1}$ refers to the average of $\theta^{m-1}$ over $\Sigma_{32}$ :

$$
\begin{gathered}
\left|\tilde{\mathbf{B}}_{2}^{m-1}\right|:=\left|\Omega_{2}\right|^{-1} \int_{\Omega_{2}}\left|\mathbf{B}_{2}\right| d x, \\
\tilde{\theta}^{m-1}:=\left|\Omega_{2}\right|^{-1} \int_{\Omega_{2}} \theta^{m-1} d x, \\
\tilde{\theta}_{s}^{m-1}:=\left|\Sigma_{32}\right|^{-1} \int_{\Sigma_{32}} \theta^{m-1} d \sigma,
\end{gathered}
$$

In terms of these quantities we define:

$$
\begin{aligned}
& \tilde{\sigma}_{2}^{m-1}:=\sigma_{2}\left(\tilde{\theta}^{m-1}\right), \quad \tilde{\xi}_{2}^{m-1}:=\xi_{2}\left(\tilde{\theta}^{m-1}, \mid \tilde{\mathbf{B}}_{2}^{m-1}\right), \\
& \tilde{q}^{m-1}:=q^{\prime}\left(\tilde{\theta}^{m-1}\right), \quad \tilde{\kappa}^{m-1}:=\kappa\left(\tilde{\theta}^{m-1}\right) .
\end{aligned}
$$

REMARK 3.1. We note that another linearization of the nonlinear B-H relationship based on Fröhlich's model has been suggested in [16]. 
The nonlinear terms from the convective and radiative heat transfer in $(2.18 \mathrm{~b})$ are linearized according to:

$$
\begin{aligned}
& h_{s}\left(\theta^{m}-\theta_{a}\right)^{\gamma} \approx h_{s}\left(\tilde{\theta}_{s}^{m-1}-\theta_{a}\right)^{\gamma}+\gamma h_{s}\left(\tilde{\theta}_{s}^{m-1}-\theta_{a}\right)^{\gamma-1}\left(\theta^{m}-\tilde{\theta}_{s}^{m-1}\right), \\
& \sigma_{\mathrm{SB}} \varepsilon\left(\left|\theta^{m}\right|^{3} \theta^{m}-\theta_{a}^{4}\right) \approx \\
& \sigma_{\mathrm{SB}} \varepsilon\left(\left|\tilde{\theta}_{s}^{m-1}\right|^{3} \tilde{\theta}_{s}^{m-1}-\theta_{a}^{4}\right)+4 \sigma_{\mathrm{SB}} \varepsilon\left(\left|\tilde{\theta}_{s}^{m-1}\right|^{2} \tilde{\theta}_{s}^{m-1}\right)\left(\theta^{m}-\tilde{\theta}_{s}^{m-1}\right) .
\end{aligned}
$$

For ease of notation we set

$$
\begin{aligned}
& \tilde{g}_{c}^{m-1}:=\gamma h_{s}\left(\tilde{\theta}_{s}^{m-1}-\theta_{a}\right)^{\gamma-1}, \quad \tilde{g}_{r}^{m-1}:=4 \sigma_{\mathrm{SB}} \varepsilon\left(\left|\tilde{\theta}_{s}^{m-1}\right|^{2} \tilde{\theta}_{s}^{m-1}\right), \\
& \tilde{g}_{T}^{m-1}:=\tilde{g}_{c}^{m-1}+\tilde{g}_{r}^{m-1}, \\
& \tilde{h}_{c}^{m-1}:=h_{s}\left(\tilde{\theta}_{s}^{m-1}-\theta_{a}\right)^{\gamma}, \quad \tilde{h}_{r}^{m-1}:=\sigma_{\mathrm{SB}} \varepsilon\left(\left|\tilde{\theta}_{s}^{m-1}\right|^{3} \tilde{\theta}_{s}^{m-1}-\theta_{a}^{4}\right), \\
& \tilde{h}_{T}^{m-1}:=\left(\tilde{g}_{c}^{m-1}+\tilde{g}_{r}^{m-1}\right) \tilde{\theta}_{s}^{m-1}-\tilde{h}_{c}^{m-1}-\tilde{h}_{r}^{m-1} .
\end{aligned}
$$

We may assume

$$
c \leq \tilde{g}_{T}^{m-1} \leq C \text { for all } 1 \leq m \leq M
$$

since we will have $\tilde{\theta}_{s}^{m-1} \geq \theta_{a}$ due to the inductive heating process.

The boundary value problem for the time discretized eddy currents equation reads

$$
\begin{aligned}
& \tilde{\sigma}^{m-1} \frac{\mathbf{A}^{m}-\mathbf{A}^{m-1}}{\Delta t}+\operatorname{curl}\left(\tilde{\xi}^{m-1} \operatorname{curl} \mathbf{A}_{m}\right)- \\
& \operatorname{grad}\left(\tilde{\xi}^{m-1} \operatorname{div} \mathbf{A}^{m}\right)=\chi_{\Omega_{1}} \mathbf{j} \mathbf{u}^{m} \cdot \boldsymbol{\psi}^{m} \text { in } D \\
& \left.\mathbf{A}^{m}\right|_{\bar{\Omega}_{i} \cap \Gamma_{i}} \wedge \mathbf{n}_{\Gamma_{i}}=-\left.\mathbf{A}^{m}\right|_{\bar{\Omega}_{3} \cap \Gamma_{3}} \wedge \mathbf{n}_{\Gamma_{i}} \\
& \mathbf{n}_{\Gamma_{i}} \wedge\left(\left.\tilde{\xi}_{i}^{m-1} \operatorname{curl} \mathbf{A}^{m}\right|_{\bar{\Omega}_{i} \cap \Gamma_{i}} \wedge \mathbf{n}_{\Gamma_{i}}\right)=\mathbf{n}_{\Gamma_{3}} \wedge\left(\left.\tilde{\xi}_{3}^{m-1} \operatorname{curl} \mathbf{A}^{m}\right|_{\Omega_{3} \cap \Gamma_{3}} \wedge \mathbf{n}_{\Gamma_{3}}\right), \\
& \left.\mathbf{n}_{\Gamma_{i}} \cdot \tilde{\xi}_{i}^{m-1} \mathbf{A}^{m}\right|_{\bar{\Omega}_{i} \cap \Gamma_{i}}=-\left.\mathbf{n}_{\Gamma_{3}} \cdot \tilde{\xi}_{3}^{m-1} \mathbf{A}^{m}\right|_{\bar{\Omega}_{i} \cap \Gamma_{3}}, \quad 1 \leq i \leq 2, \\
& \mathbf{A}^{m}=\mathbf{0} \quad \text { on } \partial D
\end{aligned}
$$

where $\boldsymbol{\psi}^{m}=\left(\psi_{1}^{m}, \psi_{2}^{m}\right)^{T}, \psi_{i}^{m}:=(\Delta t)^{-1} \int_{t_{m-1}}^{t_{m}} \cos \left(\omega_{i} t\right) d t, 1 \leq i \leq 2$.

Likewise, the boundary value problem for the time discretized heat equation takes the form

$$
\begin{aligned}
\tilde{q}^{m-1} \frac{\theta^{m}-\theta^{m-1}}{\Delta t}-\operatorname{div}\left(\tilde{\kappa}^{m-1} \operatorname{grad} \theta_{m}\right) & =\tilde{\sigma}_{2}^{m-1}\left|\frac{\mathbf{A}^{m}-\mathbf{A}^{m-1}}{\Delta t}\right|^{2} \quad \text { in } \Omega_{2}, \\
\mathbf{n}_{\Gamma_{2}} \cdot \tilde{\kappa}^{m-1} \operatorname{grad} \theta^{m}+\tilde{g}_{T}^{m-1} \theta^{m} & =\tilde{h}_{T}^{m-1} \quad \text { on } \Gamma_{2} .
\end{aligned}
$$

Setting $\mathbf{V}:=\mathbf{H}_{0}^{1}(D)^{M}, V:=H^{1}\left(\Omega_{2}\right)^{M}$, the discrete-time state space is given by $\mathbf{Y}:=$ $\mathbf{V} \times V$. The discrete-time state $\mathbf{y} \in \mathbf{Y}$ reads $\mathbf{y}=(\mathbf{A}, \theta)$ with $\mathbf{A}=\left(\mathbf{A}^{0}, \cdots, \mathbf{A}^{M}\right)^{T}, \mathbf{A}^{0}=$ $\mathbf{0}, \mathbf{A}_{m} \in \mathbf{H}_{0}^{1}(D), 1 \leq m \leq M$, and $\theta=\left(\theta^{0}, \cdots, \theta^{M}\right)^{T}, \theta^{0}=\theta_{0}, \theta^{m} \in H^{1}\left(\Omega_{2}\right), 1 \leq m \leq$ $M$. Moreover, $\mathbf{U}:=\mathbb{R}^{2 M}$ stands for the control space with controls $\mathbf{u} \in \mathbf{U}$ of the 
form $\mathbf{u}=\left(\mathbf{u}^{1}, \cdots, \mathbf{u}^{M}\right)^{T}, \mathbf{u}^{m}=\left(u_{1}^{m}, u_{2}^{m}\right)^{T} \in \mathbb{R}^{2}, 1 \leq m \leq M$.

The discrete-time optimal control problem for the inductive heating process is then given by:

(DTOC) $\inf _{(\mathbf{y}, \mathbf{u})} J_{1}(\mathbf{y})+J_{2}(\mathbf{u}), \quad J_{1}(\mathbf{y}):=\frac{1}{2}\left\|\theta^{M}-\theta_{d}\right\|_{0, \Omega_{2}^{d}}^{2}, \quad J_{2}(\mathbf{u}):=\frac{\alpha}{2} \sum_{m=1}^{M}\left|\mathbf{u}^{m}\right|^{2}$, subject to the state equations $(3.5),(3.6)$,

where $\Omega_{2}^{d} \subset \Omega_{2}$ is a subdomain of $\Omega_{2}$ around the surface $\partial \Omega_{2}$ where a desired temperature distribution $\theta_{d}$ is prescribed and $\alpha>0$ is a regularization parameter.

We will briefly address the case of control constraints $\mathbf{u} \in \mathbf{K}_{\mathbf{u}}$ where

$$
\mathbf{K}_{\mathbf{u}}:=\left\{\mathbf{u} \in \mathbb{R}^{2 M} \mid 0 \leq u_{i}^{m} \leq u_{i}^{\max }, 1 \leq i \leq 2,1 \leq m \leq M\right\}
$$

with given constants $u_{i}^{\max }>0,1 \leq i \leq 2$, and state constraints $\mathbf{K}_{\mathbf{y}}:=\mathbf{K}_{\mathbf{A}} \times K_{\theta}$ where

$$
\begin{aligned}
\mathbf{K}_{\mathbf{A}} & :=\left\{\mathbf{A}|| \mathbf{A}^{m} \mid \leq A^{\max }, 1 \leq m \leq M\right\} \\
K_{\theta} & :=\left\{\theta \mid 0 \leq \theta^{m} \leq \theta^{\max }, 1 \leq m \leq M\right\}
\end{aligned}
$$

with given constants $A^{\max }>0, \theta^{\max }>0$.

In the presence of control and state constraints the discrete-time optimal control problem reads

(DTCOC)

$$
\inf _{\mathbf{y} \in \mathbf{K}_{\mathbf{y}}, \mathbf{u} \in \mathbf{K}_{\mathbf{u}}} J_{1}(\mathbf{y})
$$

subject to the state equations $(3.5),(3.6)$,

3.2. Existence, Uniqueness, and Regularity of a Weak Solution of the Discrete-Time State Equations. The weak form of the discrete-time state equations can be written in terms of a map e : $\mathbf{V} \times V \rightarrow \mathbf{V}^{*} \times V^{*} \times \mathbf{L}^{2}(D) \times L^{2}\left(\Omega_{2}\right)$ according to

$$
\mathbf{e}(\mathbf{y}, \mathbf{u})=\left(\begin{array}{c}
\mathcal{A}_{11}^{m} \mathbf{A}^{m}-\mathcal{B}^{m} \mathbf{u}^{m}-\ell_{1}(\mathbf{Z}) \\
\mathcal{A}_{21}^{m}\left(\mathbf{A}^{m}\right)+\mathcal{A}_{22}^{m} \theta^{m}-\ell_{2}(z) \\
\mathbf{A}^{0}-\mathbf{A}_{0} \\
\theta^{0}-\theta_{0}
\end{array}\right)=\mathbf{0} .
$$

Here, $\mathcal{A}_{11}^{m}: \mathbf{V} \rightarrow \mathbf{V}^{*}$ and $\mathcal{A}_{22}^{m}: V \rightarrow V^{*}$ are the linear operators

$$
\begin{aligned}
\left\langle\mathcal{A}_{11}^{m} \mathbf{A}, \mathbf{Z}\right\rangle:= & \left(\tilde{\sigma}^{m-1} \mathbf{A}, \mathbf{Z}\right)_{0, D}+\Delta t\left(\tilde{\xi}^{m-1} \operatorname{curl} \mathbf{A}, \operatorname{curl} \mathbf{Z}\right)_{0, D}+ \\
& \Delta t\left(\tilde{\xi}^{m-1} \operatorname{div} \mathbf{A}, \operatorname{div} \mathbf{Z}\right)_{0, D}, \quad \mathbf{Z} \in \mathbf{V}, \\
\left\langle\mathcal{A}_{22}^{m} \theta, z\right\rangle:= & \left(\tilde{q}^{m-1} \theta, z\right)_{0, \Omega_{2}}+\Delta t\left(\tilde{\kappa}^{m-1} \operatorname{grad} \theta, \operatorname{grad} z\right)_{0, \Omega_{2}}+ \\
& \left(\tilde{g}_{T}^{m-1} \theta, z\right)_{0, \Sigma_{32}}, \quad z \in V,
\end{aligned}
$$

$\mathcal{A}_{21}^{m}: \mathbf{V} \rightarrow V^{*}$ is the nonlinear operator

$$
\left\langle\mathcal{A}_{21}^{m}(\mathbf{A}), z\right\rangle:=-\left(\tilde{\sigma}^{m-1}(\Delta t)^{-1}\left|\mathbf{A}-\mathbf{A}^{m-1}\right|^{2}, z\right)_{0, \Omega_{2}}, \quad z \in V,
$$

$\mathcal{B}^{m}: \mathcal{R}^{2 M} \rightarrow \mathbf{L}^{2}(D)$ is the linear operator

$$
\left(\mathcal{B}^{m}(\mathbf{u}), \mathbf{Z}\right)_{0, D}:=\Delta t\left(\chi_{\Omega_{1}} \mathbf{u}^{m} \cdot \boldsymbol{\psi}^{m} \mathbf{j}, \mathbf{Z}\right)_{0, D}, \quad \mathbf{Z} \in \mathbf{V},
$$


and the linear functionals $\ell_{1}: \mathbf{V} \rightarrow \mathbb{R}$ and $\ell_{2}: V \rightarrow \mathbb{R}$ are given by

$$
\begin{aligned}
\ell_{1}(\mathbf{Z}) & :=\left(\tilde{\sigma}^{m-1} \mathbf{A}^{m-1}, \mathbf{Z}\right)_{0, D}, \\
\ell_{2}(z) & :=-\Delta t\left(\tilde{h}_{T}^{m-1}, z\right)_{0, \Gamma_{23}}+\left(\tilde{q}^{m-1} \theta^{m-1}, z\right)_{0, \Omega_{2}} .
\end{aligned}
$$

In particular, for a given control $\mathbf{u}^{m}$, a pair $\left(\mathbf{A}^{m}, \theta^{m}\right) \in \mathbf{V} \times V$ is said to be a weak solution of (3.5) and (3.6), if it satisfies

$$
\begin{aligned}
\mathcal{A}_{11}^{m} \mathbf{A}^{m} & =\mathcal{B}^{m} \mathbf{u}^{m}+\ell_{1}, \\
\mathcal{A}_{21}^{m}\left(\mathbf{A}^{m}\right)+\mathcal{A}_{22}^{m} \theta^{m} & =\ell_{2} .
\end{aligned}
$$

The following result establishes the existence and uniqueness of a weak solution.

THEOREM 3.2. Under the assumptions on the data of the problem, for all $1 \leq$ $m \leq M$ there exists a unique weak solution $\left(\mathbf{A}^{m}, \theta^{m}\right) \in \mathbf{V} \times V$ of (3.5) and (3.6) which satisfies the energy estimates

$$
\begin{aligned}
& \left\|\operatorname{curl} \mathbf{A}^{m}\right\|_{0, D}^{2}+\left\|\operatorname{div} \mathbf{A}^{m}\right\|_{0, D}^{2}+\left\|\mathbf{A}^{m}\right\|_{0, D}^{2} \leq \\
& C\left(\left|\mathbf{u}^{m}\right|^{2}\left|\boldsymbol{\psi}_{m}\right|^{2}\|\mathbf{j}\|_{0, \Omega_{1}}^{2}+\left\|\mathbf{A}^{m-1}\right\|_{0, D}^{2}\right), \\
& \left\|\operatorname{grad} \theta^{m}\right\|_{0, \Omega_{2}}^{2}+\left\|\theta^{m}\right\|_{0, \Omega_{2}}^{2} \leq \\
& C\left(\left\|\left|\mathbf{A}^{m}-\mathbf{A}^{m-1}\right|^{2}\right\|_{0, \Omega_{2}}^{2}+\left|\tilde{h}_{T}^{m-1}\right|^{2}+\left\|\theta^{m-1}\right\|_{0, \Omega_{2}}^{2}\right) .
\end{aligned}
$$

Proof. We note that (3.14) represents a staggered system, i.e., once (3.14a) has been solved, $\mathcal{A}_{21}^{m}\left(\mathbf{A}^{m}\right)$ is known and can be shifted to the right-hand side in $(3.14 \mathrm{~b})$. Therefore, it suffices to show that the linear operators $\mathcal{A}_{11}^{m}$ and $\mathcal{A}_{22}^{m}$ are elliptic on $\mathbf{V}$ and $V$, respectively.

Observing (2.6),(2.8), it follows that the operator $\mathcal{A}_{11}^{m}$ is elliptic on $\mathbf{V}$, i.e., there exists a constant $\gamma_{1}>0$ such that

$$
\left\langle\mathcal{A}_{11}^{m} \mathbf{A}^{m}, \mathbf{A}^{m}\right\rangle \geq \gamma_{1}\left(\left\|\operatorname{curl} \mathbf{A}^{m}\right\|_{0, D}^{2}+\left\|\operatorname{div} \mathbf{A}^{m}\right\|_{0, D}^{2}+\left\|\mathbf{A}^{m}\right\|_{0, D}^{2}\right) \quad \text { for all } \mathbf{A}^{m} \in \mathbf{V}
$$

Since the functional $\ell_{1}$ is bounded on $\mathbf{V}$, the Lax-Milgram lemma guarantees the existence and uniqueness of a weak solution of (3.5). The energy estimate (3.15a) can be easily derived from (3.16) and a straightforward estimation of $\ell_{1}(\mathbf{A})$ using Young's inequality. Likewise, taking (2.15),(2.16), and (3.4) into account, we deduce the ellipticity of the operator $\mathcal{A}_{22}^{m}$ on $V$, i.e., for some constant $\gamma_{2}>0$ it holds

$$
\left\langle\mathcal{A}_{22}^{m} \theta^{m}, \theta^{m}\right\rangle \geq \gamma_{2}\left(\left\|\operatorname{grad} \theta^{m}\right\|_{0, \Omega_{2}}^{2}+\left\|\theta^{m}\right\|_{0, \Omega_{2}}^{2}\right) \quad \text { for all } \theta^{m} \in V \text {. }
$$

Since we already know that $\mathbf{A}^{m} \in \mathbf{V}, 1 \leq m \leq M$, due to the Sobolev embedding theorem $\mathbf{A}^{m}-\mathbf{A}^{m-1} \in \mathbf{L}^{4}(D)$, and hence, both functionals $\left\langle\mathcal{A}_{21}^{m}(\mathbf{A}), \cdot\right\rangle$ and $\ell_{2}(\cdot)$ are bounded on $V$. Again, the Lax-Milgram lemma asserts the existence and uniqueness of a weak solution of (3.6), and the energy estimate (3.15b) follows from (3.17) and straightforward estimation of the right-hand side.

We next show that the weak solutions $\mathbf{A}^{m}$ and $\theta^{m}, 1 \leq m \leq M$, enjoy 2-regularity which will be important to prove the existence of an optimal control. 
THEOREM 3.3. Under the assumptions on the data of the problem, for all $1 \leq$ $m \leq M$ the unique weak solutions of (3.5) and (3.6) satisfy $\mathbf{A}^{m} \in \mathbf{H}^{2}(D) \cap \mathbf{H}_{0}^{1}(D)$ and $\theta^{m} \in H^{2}\left(\Omega_{2}\right)$. In particular, we have the energy estimates

$$
\begin{aligned}
& \left\|\operatorname{curl}^{2} \mathbf{A}^{m}\right\|_{0, D}^{2}+\left\|\operatorname{grad} \operatorname{div} \mathbf{A}^{m}\right\|_{0, D}^{2}+\left\|\operatorname{curl} \mathbf{A}^{m}\right\|_{0, D}^{2}+\left\|\operatorname{div} \mathbf{A}^{m}\right\|_{0, D}^{2} \leq \text { (3.18a) } \\
& C\left(\left|\mathbf{u}^{m}\right|^{2}\left|\boldsymbol{\psi}_{m}\right|^{2}\|\operatorname{div} \mathbf{j}\|_{0, \Omega_{1}}^{2}+\left\|\operatorname{curl} \mathbf{A}^{m-1}\right\|_{0, D}^{2}+\left\|\operatorname{div} \mathbf{A}^{m-1}\right\|_{0, D}^{2}\right), \\
& \left\|\Delta \theta^{m}\right\|_{0, \Omega_{2}}^{2}+\left\|\operatorname{grad} \theta^{m}\right\|_{0, \Omega_{2}}^{2} \leq \\
& C\left(\left\|\mathbf{A}^{m}\right\|_{1, \Omega_{2}}^{4}+\left\|\mathbf{A}^{m}\right\|_{2, \Omega_{2}}^{2}+\left\|\mathbf{A}^{m-1}\right\|_{1, \Omega_{2}}^{4}+\left\|\mathbf{A}^{m-1}\right\|_{2, \Omega_{2}}^{2}+\left\|\theta^{m}\right\|_{1, \Omega_{2}}^{4}+\left\|\theta^{m-1}\right\|_{1, \Omega_{2}}^{2}\right) .
\end{aligned}
$$

Proof. The proof will be split into two parts. We will first establish 2-regularity of $\mathbf{A}^{m}$ and then that of $\theta^{m}$.

(i) We approximate $\mathbf{A}^{m}$ according to

$$
\mathbf{A}_{n}^{m}:=\sum_{i=1}^{n} a_{i}^{m} \varphi_{i}, \quad n \in \mathbb{N},
$$

by a finite sum of eigenfunctions $\varphi_{i} \in \mathbf{C}_{0}^{\infty}(D), 1 \leq i \leq n$, of the eigenvalue problem

$$
\begin{aligned}
& -\boldsymbol{\Delta} \mathbf{Z}=\operatorname{curl}^{2} \mathbf{Z}-\operatorname{grad} \operatorname{div} \mathbf{Z}=\lambda \mathbf{Z} \text { in } D, \\
& \mathbf{Z}=\mathbf{0} \text { on } \partial D .
\end{aligned}
$$

which has a countable number of increasing positive eigenvalues $\lambda_{i}, i \in \mathbb{N}$, with associated $\mathbf{L}^{2}$-orthonormal eigenfunctions. This results in an algebraic system in the unknown coefficients $a_{i}^{m}, 1 \leq i \leq n$ :

$$
\begin{aligned}
& \sum_{j=1}^{n} a_{j}^{m}\left(\left(\tilde{\sigma}^{m-1} \boldsymbol{\varphi}_{j}, \boldsymbol{\varphi}_{i}\right)_{0, D}+\Delta t\left(\tilde{\xi}^{m-1} \operatorname{curl} \boldsymbol{\varphi}_{j}, \operatorname{curl} \boldsymbol{\varphi}_{i}\right)_{0, D}+\right. \\
& \left.\Delta t\left(\tilde{\xi}^{m-1} \operatorname{div} \boldsymbol{\varphi}_{j}, \operatorname{div} \boldsymbol{\varphi}_{i}\right)_{0, D}\right)=\Delta t\left(\mathbf{u}^{m} \cdot \boldsymbol{\psi}^{m} \mathbf{j}, \boldsymbol{\varphi}_{i}\right)_{0, \Omega_{1}}+\left(\tilde{\sigma}^{m-1} \mathbf{A}^{m-1}, \boldsymbol{\varphi}_{i}\right)_{0, D},
\end{aligned}
$$

which has a unique solution. We multiply (3.19) by $\lambda_{i}$, use $-\boldsymbol{\Delta} \boldsymbol{\psi}_{i}=\lambda_{i} \boldsymbol{\psi}_{i}$, integrate by parts, then multiply by $a_{i}^{m}$, sum over all $1 \leq i \leq n$, and finally pass to the limit $n \rightarrow \infty$ to obtain

$$
\left(\tilde{\sigma}^{m-1} \mathbf{c u r l} \mathbf{A}^{m}, \operatorname{curl} \mathbf{A}^{m}\right)_{0, D}+\left(\tilde{\sigma}^{m-1} \operatorname{div} \mathbf{A}^{m}, \operatorname{div} \mathbf{A}^{m}\right)_{0, D}+
$$

$\Delta t\left(\tilde{\xi}^{m-1} \operatorname{curl}^{2} \mathbf{A}^{m}, \operatorname{curl}^{2} \mathbf{A}^{m}\right)_{0, D}+\Delta t\left(\tilde{\xi}^{m-1} \operatorname{grad} \operatorname{div} \mathbf{A}^{m}, \operatorname{grad} \operatorname{div} \mathbf{A}^{m}\right)_{0, D}=$ $\Delta t\left(\mathbf{u}^{m} \cdot \boldsymbol{\psi}^{m} \operatorname{div} \mathbf{j}, \operatorname{div} \mathbf{A}^{m}\right)_{0, \Omega_{1}}+\left(\tilde{\sigma}^{m-1} \operatorname{curl} \mathbf{A}^{m-1}, \operatorname{curl} \mathbf{A}^{m}\right)_{0, D}+$ $\left(\tilde{\sigma}^{m-1} \operatorname{div} \mathbf{A}^{m-1}, \operatorname{div} \mathbf{A}^{m}\right)_{0, D}$

where we have used curl $\mathbf{j}=\mathbf{0}$ due to $\mathbf{j}=-\operatorname{grad} h$.

The left-hand side in (3.19) can be estimated from below in view of (2.6),(2.8), whereas the right-hand side can be estimated from above by the Cauchy-Schwarz and Young's inequality, which gives rise to (3.18a) and shows $\mathbf{A}^{m} \in \mathbf{H}^{2}(D) \cap \mathbf{H}_{0}^{1}(D)$.

(ii) We proceed in the same way for $\theta^{m}$, but this time approximate by a finite number of eigenfunctions $\varphi_{i} \in C^{\infty}\left(\Omega_{2}\right)$ of the eigenvalue problem

$$
\begin{aligned}
& -\Delta z=\lambda z \quad \text { in } \Omega_{2}, \\
& \mathbf{n} \cdot \operatorname{grad} z+z=0 \text { on } \Gamma_{23} .
\end{aligned}
$$


This leads to

$\left(\tilde{q}^{m-1} \operatorname{grad} \theta^{m}, \operatorname{grad} \theta^{m}\right)_{0, D}+\Delta t\left(\tilde{\kappa}^{m-1} \operatorname{grad} \operatorname{div} \theta^{m}, \operatorname{grad} \operatorname{div} \theta^{m}\right)_{0, D}+$

$\Delta t\left(\tilde{g}_{T}^{m-1} \operatorname{grad} \theta^{m}, \operatorname{grad} \theta^{m}\right)_{0, \Gamma_{23}}=(\Delta t)^{-1}\left(\tilde{\sigma}^{m-1} \operatorname{grad}\left(\left|\mathbf{A}^{m}-\mathbf{A}^{m-1}\right|^{2}\right), \operatorname{grad} \theta^{m}\right)_{0, \Omega_{2}}$ $+\left(\tilde{q}^{m-1} \operatorname{grad} \theta^{m-1}, \operatorname{grad} \theta^{m-1}\right)_{0, \Omega_{2}}$.

In view of $(2.15),(2.16),(3.4)$, the left-hand side in (3.21) can be bounded from below by a constant times the left-hand side in the energy estimate (3.18b). The estimation of the right-hand side is a bit more elaborated. We have

$$
\operatorname{grad}\left(\left|\mathbf{A}^{m}-\mathbf{A}^{m-1}\right|^{2}=\operatorname{grad}\left(\mathbf{A}^{m} \cdot \mathbf{A}^{m}-2 \mathbf{A}^{m} \cdot \mathbf{A}^{m-1}+\mathbf{A}^{m-1} \cdot \mathbf{A}^{m-1}\right) .\right.
$$

Taking into account that

$$
\begin{aligned}
& \operatorname{grad} \mathbf{W}_{1} \cdot \mathbf{W}_{2}= \\
& \left(\mathbf{W}_{1} \cdot \operatorname{grad}\right) \mathbf{W}_{2}+\left(\mathbf{W}_{2} \cdot \operatorname{grad}\right) \mathbf{W}_{1}+\mathbf{W}_{1} \wedge \operatorname{curl} \mathbf{W}_{2}+\mathbf{W}_{2} \wedge \operatorname{curl} \mathbf{W}_{1},
\end{aligned}
$$

by an application of Hölder's inequality we find

$$
\begin{aligned}
& \left|\int_{\Omega_{2}}\left(\mathbf{A}^{m} \cdot \operatorname{grad}\right) \mathbf{A}^{m} \cdot \operatorname{grad} \theta^{m} d x\right|= \\
& \left|\int_{\Omega_{2}} \mathbf{A}^{m} \cdot\left(\boldsymbol{\nabla}^{T} \mathbf{A}^{m} \operatorname{grad} \theta^{m}\right) d x\right| \leq\left\|\mathbf{A}^{m}\right\|_{0,4, \Omega_{2}}\left\|\mathbf{A}^{m}\right\|_{1,4, \Omega_{2}}\left\|\theta^{m}\right\|_{1,2, \Omega_{2}} .
\end{aligned}
$$

By the Sobolev embedding theorem, $\mathbf{H}^{1}\left(\Omega_{2}\right)$ and $\mathbf{H}^{2}\left(\Omega_{2}\right)$ are continuously embedded in $\mathbf{L}^{4}\left(\Omega_{2}\right)$ and $\mathbf{W}^{1,4}\left(\Omega_{2}\right)$, whence

$$
\left\|\mathbf{A}^{m}\right\|_{0,4, \Omega_{2}}\left\|\mathbf{A}^{m}\right\|_{1,4, \Omega_{2}}\left\|\theta^{m}\right\|_{1,2, \Omega_{2}} \leq C\left\|\mathbf{A}^{m}\right\|_{1, \Omega_{2}} \mid \theta^{m}\left\|_{1, \Omega_{2}}\right\| \mathbf{A}^{m} \|_{2, \Omega_{2}} .
$$

Now, applying the generalized Young inequality

$$
\prod_{i=1}^{3} a_{i} \leq C\left(a_{1}^{1 / p_{1}}+a_{2}^{1 / p_{2}}+a_{3}^{1 / p_{3}}\right), \quad \sum_{i=1}^{3} p_{i}=1
$$

with $p_{1}=p_{2}=1 / 4$ and $p_{3}=1 / 2,(3.24)$ implies

$$
\left\|\mathbf{A}^{m}\right\|_{1, \Omega_{2}}\left\|\theta^{m}\right\|_{1, \Omega_{2}}\left\|\mathbf{A}^{m}\right\|_{2, \Omega_{2}} \leq C\left(\left\|\mathbf{A}^{m}\right\|_{1, \Omega_{2}}^{4}+\left\|\theta^{m}\right\|_{1, \Omega_{2}}^{4}+\left\|\mathbf{A}^{m}\right\|_{2, \Omega_{2}}^{2}\right),
$$

such that from (3.23) we deduce

$$
\left|\int_{\Omega_{2}}\left(\mathbf{A}^{m} \cdot \operatorname{grad}\right) \mathbf{A}^{m} \cdot \operatorname{grad} \theta^{m} d x\right| \leq C\left(\left\|\mathbf{A}^{m}\right\|_{1, \Omega_{2}}^{4}+\left\|\theta^{m}\right\|_{1, \Omega_{2}}^{4}+\left\|\mathbf{A}^{m}\right\|_{2, \Omega_{2}}^{2}\right) .
$$


Likewise, we obtain the upper bounds

$$
\begin{aligned}
& \mid \int_{\Omega_{2}}\left(\mathbf{A}^{m} \wedge \operatorname{curl} \mathbf{A}^{m} \cdot \operatorname{grad} \theta^{m} d x \mid \leq C\left(\left\|\mathbf{A}^{m}\right\|_{1, \Omega_{2}}^{4}+\left\|\theta^{m}\right\|_{1, \Omega_{2}}^{4}+\left\|\mathbf{A}^{m}\right\|_{2, \Omega_{2}}^{2}\right),\right. \\
& \left|\int_{\Omega_{2}}\left(\mathbf{A}^{m-1} \cdot \operatorname{grad}\right) \mathbf{A}^{m} \cdot \operatorname{grad} \theta^{m} d x\right| \leq C\left(\left\|\mathbf{A}^{m-1}\right\|_{1, \Omega_{2}}^{4}+\left\|\theta^{m}\right\|_{1, \Omega_{2}}^{4}+\left\|\mathbf{A}^{m}\right\|_{2, \Omega_{2}}^{2}\right), \\
& \mid \int_{\Omega_{2}}\left(\mathbf{A}^{m-1} \wedge \operatorname{curl} \mathbf{A}^{m} \cdot \operatorname{grad} e^{m} d x \mid \leq C\left(\left\|\mathbf{A}^{m-1}\right\|_{1, \Omega_{2}}^{4}+\left\|\theta^{m}\right\|_{1, \Omega_{2}}^{4}+\left\|\mathbf{A}^{m}\right\|_{2, \Omega_{2}}^{2}\right),\right. \\
& \left|\int_{\Omega_{2}}\left(\mathbf{A}^{m} \cdot \operatorname{grad}\right) \mathbf{A}^{m-1} \cdot \operatorname{grad} e^{m} d x\right| \leq C\left(\left\|\mathbf{A}^{m}\right\|_{1, \Omega_{2}}^{4}+\left\|\theta^{m}\right\|_{1, \Omega_{2}}^{4}+\left\|\mathbf{A}^{m-1}\right\|_{2, \Omega_{2}}^{2}\right), \\
& \mid \int_{\Omega_{2}}\left(\mathbf{A}^{m} \wedge \mathbf{c u r l} \mathbf{A}^{m-1} \cdot \operatorname{grad} e^{m} d x \mid \leq C\left(\left\|\mathbf{A}^{m}\right\|_{1, \Omega_{2}}^{4}+\left\|\theta^{m}\right\|_{1, \Omega_{2}}^{4}+\left\|\mathbf{A}^{m-1}\right\|_{2, \Omega_{2}}^{2}\right) .\right.
\end{aligned}
$$

Summarizing the preceding estimates and (3.25), for the first term on the right-hand side in (3.21) it follows that

$$
\begin{aligned}
& \left|(\Delta t)^{-1}\left(\tilde{\sigma}^{m-1} \operatorname{grad}\left(\left|\mathbf{A}^{m}-\mathbf{A}^{m-1}\right|^{2}\right), \operatorname{grad} \theta^{m}\right)_{0, \Omega_{2}}\right| \leq \\
& C\left(\left\|\mathbf{A}^{m}\right\|_{1, \Omega_{2}}^{4}+\left\|\mathbf{A}^{m}\right\|_{2, \Omega_{2}}^{2}+\left\|\mathbf{A}^{m-1}\right\|_{1, \Omega_{2}}^{4}+\left\|\mathbf{A}^{m-1}\right\|_{2, \Omega_{2}}^{2}+\left\|\theta^{m}\right\|_{1, \Omega_{2}}^{4}\right) .
\end{aligned}
$$

For the second term on the right-hand side in (3.21) we obtain

$$
\left|\left(\tilde{q}^{m-1} \operatorname{grad} \theta^{m-1}, \operatorname{grad} \theta^{m-1}\right)_{0, \Omega_{2}}\right| \leq C\left\|\theta^{m-1}\right\|_{1, \Omega_{2}}^{2} .
$$

Combining (3.26),(3.27) we deduce the upper bound in (3.18b) which shows that $\theta^{m} \in H^{2}\left(\Omega_{2}\right)$.

\section{Existence of a Solution of the Discrete-Time Optimal Control Prob-}

lem. The preceding subsection provided all prerequisites to prove the existence of a solution of the discrete-time optimal control problem (DTOC).

THEOREM 4.1. Under the assumptions on the data of the problem, the discretetime optimal control problem (DTOC) has a solution.

Proof. We denote by $\mathbf{S}: \mathbb{R}^{M} \rightarrow \mathbf{V} \times V$ the control-to-state map which assigns to a control vector $\mathbf{u}=\left(\mathbf{u}^{1}, \cdots, \mathbf{u}^{M}\right)^{T}$ the unique weak solutions $\mathbf{A}^{m} \in \mathbf{V}, \theta^{m} \in V, 1 \leq$ $m \leq M$, of the state equations (3.5),(3.6). Then, the control-reduced formulation of (DTOC) reads

$$
\inf _{\mathbf{u}} \hat{J}(\mathbf{u}), \quad \hat{J}(\mathbf{u}):=J_{1}(\mathbf{S}(\mathbf{u}))+J_{2}(\mathbf{u})
$$

Let $\left\{\mathbf{u}^{n}\right\}_{\mathbb{N}}, \mathbf{u}^{n}=\left(\mathbf{u}^{1, n}, \cdots, \mathbf{u}^{M, n}\right)^{T} \in \mathbb{R}^{2 M}, n \in \mathbb{N}$, be a minimizing sequence. Due to the coerciveness of the objective functional in $\mathbf{u}$, the minimizing sequence is bounded and hence, there exist a subsequence $\mathbb{N}^{\prime} \subset \mathbb{N}$ and a vector $\mathbf{u}^{*} \in \mathbb{R}^{2 M}$ such that $\mathbf{u}^{n} \rightarrow$ $\mathbf{u}^{*}\left(\mathbb{N}^{\prime} \ni n \rightarrow \infty\right)$. Since the objective functional is lower semicontinuous, it follows that $\mathbf{u}^{*}$ is a minimizer. Let now $\mathbf{y}^{n}=\mathbf{S}\left(\mathbf{u}^{n}\right)$ with $\mathbf{y}^{n}=\left(\mathbf{A}^{n}, \theta^{n}\right) \in \mathbf{V} \times V, n \in \mathbb{N}^{\prime}$, where $\mathbf{A}^{n}=\left(\mathbf{A}^{0, n}, \cdots, \mathbf{A}^{M, n}\right)^{T}, \mathbf{A}^{0, n}=\mathbf{0}$ and $\mathbf{A}^{m, n} \in \mathbf{H}_{0}^{1}(D), 1 \leq m \leq M$, and $\theta^{n}=\left(\theta^{0, n}, \cdots, \theta^{M, n}\right)^{T}, \theta^{0, n}=\theta_{0}$, and $\theta^{m, n} \in H^{1}\left(\Omega_{2}\right), 1 \leq m \leq M$, are the unique weak solutions of the state equations (3.5),(3.6) for the control $\mathbf{u}^{n} \in \mathbb{R}^{2 M}$. It follows from the results of section 3 that for each $1 \leq m \leq M$ the sequences $\left\{\mathbf{A}^{m, n}\right\}_{n \in \mathbb{N}^{\prime}}$ and 
$\left\{\theta^{m, n}\right\}_{n \in \mathbb{N}^{\prime}}$ are bounded in $\mathbf{H}^{2}(D) \cap \mathbf{H}_{0}^{1}(D)$ and $H^{2}\left(\Omega_{2}\right)$, respectively. Consequently, there exist another subsequence $\mathbb{N}^{\prime \prime} \subset \mathbb{N}^{\prime}$ and functions $\mathbf{A}^{m, *} \in \mathbf{H}^{2}(D) \cap \mathbf{H}_{0}^{1}(D)$ and $\theta^{m, *} \in H^{2}\left(\Omega_{2}\right)$ such that $\mathbf{A}^{m, n} \rightarrow \mathbf{A}^{m, *}$ in $\mathbf{H}^{2}(D) \cap \mathbf{H}_{0}^{1}(D)$ and $\theta^{m, n} \rightarrow \theta^{m, *}$ in $H^{2}\left(\Omega_{2}\right)$ for $\mathbb{N}^{\prime \prime} \ni n \rightarrow \infty$. Due to the Rellich-Kondrachov embedding theorems, we have $\mathbf{A}^{m, n} \rightarrow \mathbf{A}^{m, *}$ in $\mathbf{H}_{0}^{1}(D)$ and $\theta^{m, n} \rightarrow \theta^{m, *}$ in $H^{1}\left(\Omega_{2}\right)$. This implies as well strong convergence of the averages $\left|\tilde{\mathbf{B}}_{2}^{m-1, n}\right|, \tilde{\theta}^{m-1, n}, \tilde{\theta}_{s}^{m-1, n}$, and thus strong convergence of the coefficients $\tilde{\sigma}_{2}^{m-1, n}, \widetilde{\xi}_{2}^{m-1, n}, \tilde{q}^{m-1, n}, \tilde{\kappa}^{m-1, n}$, and of $\tilde{g}_{T}^{m-1, n}, \tilde{h}_{T}^{m-1, n}$. Passing to the limit in the equations satisfied by $\mathbf{A}^{m, n}$ and $\theta^{m, n}$, it follows that the pair $\left(\mathbf{A}^{*}, \theta^{*}\right)$ is the unique weak solution the state equations $(3.5),(3.6)$ for the control $\mathbf{u}^{*} \in \mathbf{U}$, i.e., $\mathbf{y}^{*}=\mathbf{S}\left(\mathbf{u}^{*}\right)$.

Likewise, we can prove the existence of a solution in case of the control and state constrained discrete-time optimal control problem (DTCOC:

THEOREM 4.2. In addition to the assumptions of Theorem 4.1 suppose that

$$
\mathbf{S}\left(\mathbf{K}_{\mathbf{u}}\right) \subset \mathbf{K}_{\mathbf{y}}
$$

which can be guaranteed by a proper choice of $A^{\max }$ and $\theta^{\max }$. Then, the control and state constrained discrete-time optimal control problem (DTCOC) has a solution.

Proof. The proof follows the same pattern as the proof of Theorem 4.1. The minimizing sequence $\left\{\mathbf{u}^{n}\right\}_{\mathbb{N}}$ is bounded due to $\mathbf{u}^{n} \in \mathbf{K}_{\mathbf{u}}, n \in \mathbb{N}$ and hence, there exist a subsequence $\mathbb{N}^{\prime} \subset \mathbb{N}$ and a vector $\mathbf{u}^{*} \in \mathbb{R}^{2 M}$ such that $\mathbf{u}^{n} \rightarrow \mathbf{u}^{*}\left(\mathbb{N}^{\prime} \ni n \rightarrow \infty\right)$. Since $\mathbf{K}_{\mathbf{u}}$ is closed, we have $\mathbf{u}^{*} \in \mathbf{K}_{\mathbf{u}}$. The rest of the proof is the same as before, and at the end we deduce $\mathbf{y}^{*}=\left(\mathbf{A}^{*}, \theta^{*}\right) \in \mathbf{K}_{\mathbf{y}}$ due to (4.2).

5. The Discrete-Time Optimality System. The optimality conditions invoke an adjoint state

$$
\begin{aligned}
& \mathbf{p}=(\mathbf{W}, \Theta) \text { with } \mathbf{W}:=\left(\mathbf{W}^{0}, \cdots, \mathbf{W}^{M}\right), \mathbf{W}^{m} \in \mathbf{V}, 0 \leq m \leq M, \text { and } \\
& \Theta:=\left(\Theta^{0}, \cdots, \Theta^{M}\right) \text { with } \Theta^{m} \in V, 0 \leq m \leq M-1, \Theta^{M} \in L^{2}\left(\Omega_{2}\right) .
\end{aligned}
$$

In terms of the map e $:=\mathbf{Y} \times \mathbf{U} \rightarrow \mathbf{Y}^{*} \times \mathbf{L}^{2}(D) \times L^{2}\left(\Omega_{2}\right)$, we have [29]

$$
\begin{aligned}
& \mathbf{e}_{\mathbf{y}}(\mathbf{y}, \mathbf{u})^{*} \mathbf{p}=J_{\mathbf{y}}(\mathbf{y}, \mathbf{u}), \\
& \mathbf{e}_{\mathbf{u}}(\mathbf{y}, \mathbf{u})^{*} \mathbf{p}+J_{\mathbf{u}}(\mathbf{y}, \mathbf{u})=\mathbf{0},
\end{aligned}
$$

where $\mathbf{e}_{\mathbf{y}}(\mathbf{y}, \mathbf{u})^{*}$ and $\mathbf{e}_{\mathbf{u}}(\mathbf{y}, \mathbf{u})^{*}$ stand for the adjoints of the Fréchet derivatives of $\mathbf{e}$ with respect to $\mathbf{y}$ and $\mathbf{u}$ at $(\mathbf{y}, \mathbf{u})$ and $J_{\mathbf{y}}(\mathbf{y}, \mathbf{u}), J_{\mathbf{u}}(\mathbf{y}, \mathbf{u})$ are the partial Gâteaux derivatives of the objective functional with respect to $\mathbf{y}$ and $\mathbf{u}$ at $(\mathbf{y}, \mathbf{u})$.

The discrete-time adjoint state equations (5.1a) read

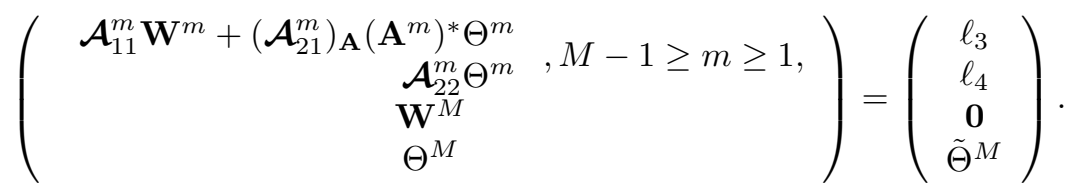

Here, $\tilde{\Theta}^{M}=\theta_{d}-\theta^{M}$ in $\Omega_{2}^{d}$ and $\tilde{\Theta}^{M}=0$ in $\Omega_{2} \backslash \bar{\Omega}_{2}^{d}$. Moreover, $\left(\mathcal{A}_{21}^{m}\right)_{\mathbf{A}}\left(\mathbf{A}^{m}\right)^{*}$ is the adjoint of the Fréchet derivative of $\mathcal{A}_{21}^{m}$ with respect to $\mathbf{A}$ at $\mathbf{A}^{m}$

$$
\left\langle\left(\mathcal{A}_{21}^{m}\right)_{\mathbf{A}}\left(\mathbf{A}^{m}\right)^{*} \Theta^{m}, \mathbf{Z}\right\rangle=-2(\Delta t)^{-1}\left(\tilde{\sigma}_{2}^{m-1} \Theta^{m}\left(\mathbf{A}^{m}-\mathbf{A}^{m-1}\right), \mathbf{Z}\right)_{0, \Omega_{2}},
$$

and the functionals $\ell_{3}: \mathbf{V} \rightarrow \mathbb{R}$ and $\ell_{4}: V \rightarrow \mathbb{R}$ are given by

$$
\ell_{3}(\mathbf{Z}):=\left(\tilde{\sigma}^{m} \mathbf{W}^{m+1}, \mathbf{Z}\right)_{0, D}, \quad \ell_{4}(z):=\left(\tilde{q}^{m} \Theta^{m+1}, z\right)_{0, \Omega_{2}} .
$$


In view of $J_{2}$ in (DTOC) and (3.9), the optimality condition (5.1b) takes the form

$$
\psi_{i}^{m}\left(\mathbf{j}, \mathbf{W}^{m}\right)_{0, \Omega_{1}}+\alpha u_{i}^{m}=0, \quad 1 \leq i \leq 2
$$

We have thus established the optimality system for the discrete-time optimal control problem (DTOC):

Theorem 5.1. Let $(\mathbf{y}, \mathbf{u}) \in \mathbf{Y} \times \mathbf{U}$ with $\mathbf{y}=(\mathbf{A}, \theta) \in \mathbf{V} \times V$ and $\mathbf{u} \in \mathbf{U}$ be a solution of the discrete-time optimal control problem (DTOC). Then, there exists an optimal discrete-time adjoint state $\mathbf{p} \in \mathbf{Y}$ with $\mathbf{p}=(\mathbf{W}, \Theta) \in \mathbf{V} \times V$ such that the triple $(\mathbf{y}, \mathbf{p}, \mathbf{u})$ satisfies the discrete-time state equations (3.9), the discrete-time adjoint equations (5.2), and the gradient equation (5.3).

The discrete-time adjoint state equations (5.2) are solved backward in time from $m=$ $M-1$ to $m=1$. They represent a staggered system such that for each $M-1 \geq m \geq 1$ the equation for the discrete-time adjoint temperature $\Theta^{m}$ is solved first followed by the equation for the discrete-time adjoint magnetic vector potential $\mathbf{W}^{m}$.

REMARK 5.2. Rewriting (5.2) in the strong form

$$
\begin{aligned}
& -\left(\tilde{\sigma}^{m-1} \frac{\mathbf{W}^{m+1}-\mathbf{W}^{m}}{\Delta t}+\frac{\tilde{\sigma}^{m}-\tilde{\sigma}^{m-1}}{\Delta t} \mathbf{W}^{m}\right)+\operatorname{curl}\left(\tilde{\xi}^{m-1} \operatorname{curl} \mathbf{W}_{m}\right)- \\
& \operatorname{grad}\left(\tilde{\xi}^{m-1} \operatorname{div} \mathbf{W}^{m}\right)=2 \chi_{\Omega_{2}}(\Delta t)^{-2}\left(\tilde{\sigma}_{2}^{m-1} \Theta^{m}\left(\mathbf{A}^{m}-\mathbf{A}^{m-1}\right) \quad \text { in } D,\right. \\
& \left.\mathbf{W}^{m}\right|_{\bar{\Omega}_{i} \cap \Gamma_{i}} \wedge \mathbf{n}_{\Gamma_{i}}=-\left.\mathbf{W}^{m}\right|_{\bar{\Omega}_{3} \cap \Gamma_{3}} \wedge \mathbf{n}_{\Gamma_{i}} \\
& \mathbf{n}_{\Gamma_{i 3}} \wedge\left(\left.\tilde{\xi}_{i}^{m-1} \operatorname{curl} \mathbf{W}^{m}\right|_{\bar{\Omega}_{i} \cap \Gamma_{i 3}} \wedge \mathbf{n}_{\Gamma_{i 3}}\right)=\mathbf{n}_{\Gamma_{3 i}} \wedge\left(\left.\tilde{\xi}_{3}^{m-1} \operatorname{curl} \mathbf{W}^{m}\right|_{\bar{\Omega}_{3} \cap \Gamma_{3 i}} \wedge \mathbf{n}_{\Gamma_{3 i}}\right), \\
& \left.\mathbf{n}_{\Gamma_{i 3}} \cdot \tilde{\xi}_{i}^{m-1} \mathbf{W}^{m}\right|_{\bar{\Omega}_{i} \cap \Gamma_{i 3}}=-\left.\mathbf{n}_{\Gamma_{3 i}} \cdot \tilde{\xi}_{3}^{m-1} \mathbf{W}^{m}\right|_{\bar{\Omega}_{i} \cap \Gamma_{3 i}}, \quad 1 \leq i \leq 2, \\
& \mathbf{W}^{m}=\mathbf{0} \text { on } \partial D,
\end{aligned}
$$

and

$$
\begin{aligned}
-\left(\tilde{q}^{m} \frac{\Theta^{m+1}-\Theta^{m}}{\Delta t}+\frac{\tilde{q}^{m}-\tilde{q}^{m-1}}{\Delta t} \Theta^{m}\right)-\operatorname{div}\left(\tilde{\kappa}^{m-1} \operatorname{grad} \Theta_{m}\right)=0 & \text { in } \Omega_{2}, \\
\mathbf{n}_{\Gamma_{23}} \cdot \tilde{\kappa}^{m-1} \operatorname{grad} \Theta^{m}+\tilde{g}_{T}^{m-1} \Theta^{m}=0 & \text { on } \Gamma_{23},
\end{aligned}
$$

where $\mathbf{W}^{M}=\mathbf{0}$ and $\Theta^{M}=\tilde{\Theta}^{M}$, it can be interpreted as the time-discretized version of a backward in time eddy currents equation and a backward in time heat equation.

REMARK 5.3. The optimality system of the discrete-time optimal control problem (DTOC) can be solved by the gradient method. Starting from an initial control $\mathbf{u}_{0}$, we first solve the discrete-time state equations (3.9), followed by the discrete-time adjoint state equations (5.2), and then update the control via (5.3) combined with an Armijo line search (cf., e.g., [15]).

Let us briefly present the optimality system in case of the control and state constrained discrete-time optimal control problem (DTCOC). Denoting by $I_{\mathbf{K}_{\mathbf{u}}}, I_{\mathbf{K}_{\mathbf{y}}}$ the indicator 
functions of the set $\mathbf{K}_{\mathbf{u}}$ of control constraints and of the set $\mathbf{K}_{\mathbf{y}}$ of state constraints, the unconstrained control reduced form of (DTCOC) is given by

$$
\inf _{\mathbf{u}} J_{1}(\mathbf{S}(\mathbf{u}))+I_{\mathbf{K}_{\mathbf{u}}}(\mathbf{u})+I_{\mathbf{K}_{\mathbf{y}}}(\mathbf{S u}) .
$$

The optimality condition for (5.6) reads

$$
\mathbf{0} \in \mathbf{S}_{\mathbf{u}}(\mathbf{u})^{*} J_{1, \mathbf{y}}(\mathbf{S}(\mathbf{u}))+\partial\left(I_{\mathbf{K}_{\mathbf{y}}} \circ \mathbf{S}\right)(\mathbf{u})+\partial I_{\mathbf{K}_{\mathbf{u}}}(\mathbf{u})
$$

where $\mathbf{S}_{\mathbf{u}}(\mathbf{u}) \in \mathcal{L}(\mathbf{U}, \mathbf{Y})$ is the Fréchet derivative of $\mathbf{S}$ at $\mathbf{u}$ and $\partial\left(I_{\mathbf{K}_{\mathbf{y}}} \circ \mathbf{S}\right)(\mathbf{u}), \partial I_{\mathbf{K}_{\mathbf{u}}}(\mathbf{u})$ are the subdifferentials of the indicator functions of $I_{\mathbf{K}_{\mathbf{y}}} \circ \mathbf{S}$ and of $I_{\mathbf{K}_{\mathbf{u}}}$ at $\mathbf{u}$. Since the states $\mathbf{A}=\left(\mathbf{A}^{1}, \cdots, \mathbf{A}^{M}\right)^{T}$ and $\theta=\left(\theta^{1}, \cdots, \theta^{M}\right)^{T}$ are continuous due to Theorem 3.3 and $\mathbf{S}(\overline{\mathbf{u}})=\left(\mathbf{0}, \theta_{0}\right) \in \operatorname{int}\left(\mathbf{K}_{\mathbf{y}}\right)$ for $\overline{\mathbf{u}}=\mathbf{0}$, the control $\overline{\mathbf{u}}=\mathbf{0}$ is a Slater point and hence, it holds (cf., e.g., [10]):

$$
\partial\left(I_{\mathbf{K}_{\mathbf{y}}} \circ \mathbf{S}\right)(\mathbf{u})=\mathbf{S}_{\mathbf{u}}(\mathbf{u})^{*} \partial I_{\mathbf{K}_{\mathbf{y}}}(\mathbf{S}(\mathbf{u})) .
$$

We note that $\partial I_{\mathbf{K}_{\mathbf{A}}}(\mathbf{A}) \subset 2^{\mathcal{M}(D)}, \mathcal{M}(D)=\mathbf{C}(D)^{*}$, and $\partial I_{\mathbf{K}_{\theta}}(\theta) \subset 2^{\mathcal{M}\left(\Omega_{2}\right)}, \mathcal{M}\left(\Omega_{2}\right)=$ $C\left(\Omega_{2}\right)^{*}$. Hence, there exist a vector-valued regular Borel measure $\boldsymbol{\lambda}_{1} \in \mathcal{M}(D)$, a regular Borel measure $\lambda_{2} \in \mathcal{M}\left(\Omega_{2}\right)$, and $\boldsymbol{\mu} \in I_{\mathbf{K}_{\mathbf{u}}}(\mathbf{u}) \subset 2^{\mathbb{R}^{2 M}}$ such that with $\boldsymbol{\lambda}=\left(\boldsymbol{\lambda}_{1}, \lambda_{2}\right)$ the optimality system for (DTCOC) can be written as

$$
\begin{aligned}
& \mathbf{e}_{\mathbf{y}}(\mathbf{y}, \mathbf{u})^{*} \mathbf{p}=J_{1, \mathbf{y}}(\mathbf{y})+\boldsymbol{\lambda} \\
& \mathbf{e}_{\mathbf{u}}(\mathbf{y}, \mathbf{u})^{*} \mathbf{p}+\boldsymbol{\mu}=\mathbf{0}
\end{aligned}
$$

Since the Borel measures occur on the right-hand side of the discrete-time adjoint system, we cannot expect $\mathbf{p}=(\mathbf{W}, \Theta) \in \mathbf{V} \times V$, but rather $\mathbf{W}^{m} \in \mathbf{W}_{0}^{1, s}(D)$ and $\Theta^{m} \in W^{1, s}\left(\Omega_{2}\right)$ for some $s<2[4]$. In order to derive a discrete-time adjoint system like (5.2) in the unconstrained case, we redefine the operators $\mathcal{A}_{11}^{m}, \mathcal{A}_{21}$, and $\mathcal{A}_{22}^{m}$ as operators $\mathcal{A}_{11}^{m}: \mathbf{W}_{0}^{1, r}(D) \rightarrow \mathbf{W}^{-1, r}(D), \mathcal{A}_{21}^{m}: \mathbf{W}_{0}^{1, r}(D) \rightarrow W^{-1, r}\left(\Omega_{2}\right)$, and $\mathcal{A}_{22}^{m}:$ $W^{1, r}\left(\Omega_{2}\right) \rightarrow W^{-1, r}\left(\Omega_{2}\right)$ for $r>3$ such that $\left(\mathcal{A}_{21}^{m}\right) \mathbf{A}\left(\mathbf{A}^{m}\right)^{*} \in \mathcal{L}\left(W^{1, s}\left(\Omega_{2}\right), \mathbf{W}^{-1, s}(D)\right)$, where $s$ is conjugate to $r$. Likewise, the functionals $\ell_{3}$ and $\ell_{4}$ have to be redefined as functionals on $\mathbf{W}_{0}^{1, r}(D)$ and $W^{1, r}\left(\Omega_{2}\right)$, i.e., $\ell_{3} \in \mathbf{W}^{-1, s}(D)$ and $\ell_{4} \in W^{-1, s}\left(\Omega_{2}\right)$. In more explicit form, the discrete-time adjoint state equations for (DTCOC) read

$$
\left(\begin{array}{c}
\left(\mathcal{A}_{11}^{m}\right)^{*} \mathbf{W}^{m}+\left(\mathcal{A}_{21}^{m}\right)_{\mathbf{A}}\left(\mathbf{A}^{m}\right)^{*} \Theta^{m} \\
\left(\mathcal{A}_{22}^{m}\right)^{*} \Theta^{m} \\
\mathbf{W}^{M} \\
\Theta^{M}
\end{array}\right)=\left(\begin{array}{c}
\ell_{3}+\boldsymbol{\lambda}_{1} \\
\ell_{4}+\lambda_{2} \\
\mathbf{0} \\
\tilde{\Theta}^{M}
\end{array}\right)
$$

The optimality system can be solved by a primal-dual active set strategy based on a Moreau-Yosida approximation of the subdifferentials of the indicator functions which can be implemented as a semi-smooth Newton method (cf., e.g., [12]).

6. Numerical Results. For the spatial discretization of the optimality system for the discrete-time optimal control problem (DTOC) and the optimality system for the discrete-time control and state constrained optimal control problem (DTCOC) we may use continuous, piecewise linear finite elements with respect to a shape-regular family of geometrically conforming simplicial triangulations $\mathcal{T}_{h}(D)$ of the computational domain $D$ which aligns with the partition of $D$ into the subdomains $\Omega_{i}, 1 \leq i \leq 3$, in the sense that $\mathcal{T}_{h}(D)$ induces geometrically conforming simplicial triangulations $\mathcal{T}_{h}\left(\Omega_{i}\right), 1 \leq i \leq 3$. 


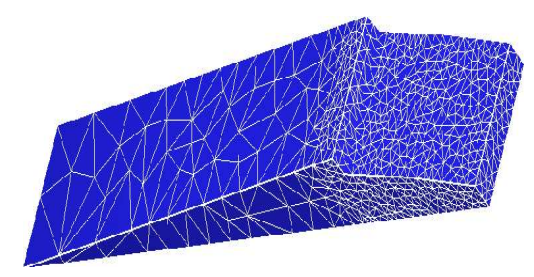

FIG. 6.1. Triangulation of a quarter-tooth of the workpiece.

Following Remark 2.2, an alternative is to use continuous, piecewise linear finite elements only for the discretization of the trial and test functions $\varphi, \psi \in H_{0}^{1}\left(\Omega_{3}\right)$ in the weakly regularized magnetic vector potential equations $(2.14 \mathrm{a}),(2.14 \mathrm{~b})$ as well as for the temperature $\theta$, whereas to use the lowest order edge elements of Nédélec's first family $[20,21]$ for the spatial discretization of $\mathbf{A}_{i}, 1 \leq i \leq 3$. The numerical results reported in this section are based on the latter approach applied to the optimality system for the discrete-time optimal control problem (DTOC) without considering the convective and radiative heat transfer across $\partial \Omega_{2}$. We have further assumed

- a simplified geometric configuration with the radii $r_{b}$ and $r_{t}$ of the base and the tip circle of the workpiece given by $r_{b}=18.15 \mathrm{~mm}, r_{t}=23.85 \mathrm{~mm}$, the distance $d$ between the tip circle and the coil by $d=1.15 \mathrm{~mm}$, the radii of the coil by $r_{i}:=25 \mathrm{~mm}$ and $r_{o}:=33 \mathrm{~mm}$, and the radius $r_{D}$ of the entire computational domain by $r_{D}=64 \mathrm{~mm}$. The thickness of the workpiece and the coil has been chosen as $8 \mathrm{~mm}$,

- a high frequency of $\omega_{1}=100 \mathrm{kHz}$ with a maximum voltage of $U_{1}=100 \mathrm{~V}$ and a medium frequency of $\omega_{2}=20 \mathrm{kHz}$ with a maximum voltage of $U_{2}=50 \mathrm{~V}$,

- a preheated workpiece with an initial uniform temperature of $\theta_{0}=500{ }^{\circ} \mathrm{C}$ and a desired temperature of $\theta_{d}=800^{\circ} C$ in $\Omega_{2}^{d}:=\left\{x \in \Omega_{2} \mid \operatorname{dist}\left(x, \partial \Omega_{2}\right)<\right.$ $17 \mathrm{~mm}\}$,

- a total duration of $T=2.0 \mathrm{~s}$ of the inductive heating process.

The actual discretization in time has been done using a time step size of $\delta t=1.25$. $10^{-6} \mathrm{~s}$ for the eddy currents equations in $\Omega_{1}$ and $\Omega_{2}$ and a time step size of $\Delta t=$ $0.04 s$ for the heat equation. Consequently, the freezing of the coefficients in the eddy currents equations in $\Omega_{2}$ has been done with respect to time intervals of length $\Delta t$. The eddy currents equations have been iterated until a nearly periodic solution emerged which has then be used to compute the source term in the heat equation by time averaging. For discretization in space, we have used a geometrically conforming triangulation with an average mesh width of $h=0.8 \mathrm{~mm}$ for $\Omega_{1}$ (coil), $h=0.4 \mathrm{~mm}$ in $\Omega_{2}^{d}$ (fine part of the workpiece), $h=1.5 \mathrm{~mm}$ for $\Omega_{2} \backslash \Omega_{2}^{d}$ (coarse part of the workpiece), and $h=4 \mathrm{~mm}$ for $\Omega_{3}$ (air). The triangulation of a quarter-tooth of the workpiece is displayed in Figure 6.1.

The optimality system for the fully discrete optimal control problem (DTOC) has been solved by a gradient method with Armijo line search using an initial control $\mathbf{u}^{(0)}=\left(u_{1}^{(0)}, u_{2}^{(0)}\right)^{T}$ with all components of the initial high frequency control $u_{1}^{(0)}$ set to 0.1 and all components of the initial medium frequency control $u_{2}^{(0)}$ set to 1.0 . 

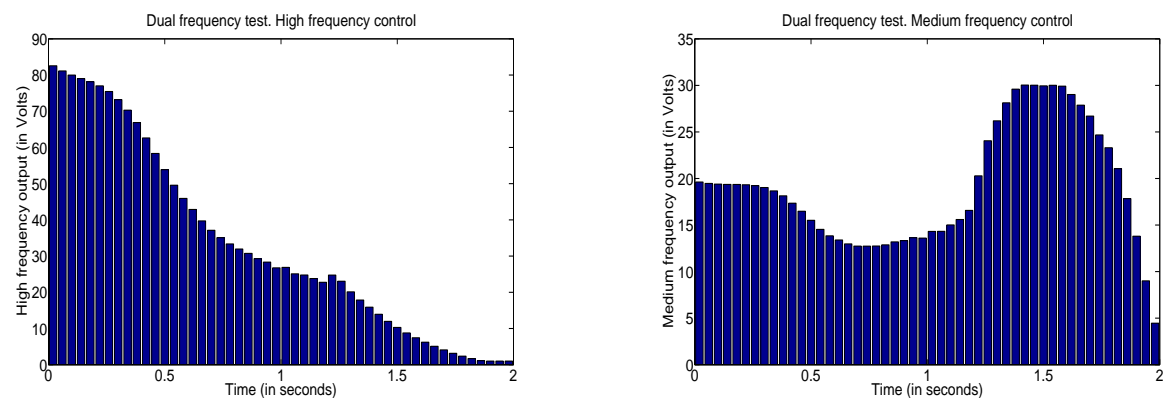

FIG. 6.2. Optimal output voltages $u_{1} U_{1}$ (high frequency; left) and $u_{2} U_{2}$ (medium frequency; right).

Figure 6.2 displays the computed optimal output voltages $u_{1} U_{1}$ for the high frequency (left) and $u_{2} U_{2}$ for the medium frequency (right) at the time instances $t_{m}=m \Delta t, 0 \leq$ $m \leq M=50$. It turns out that it is optimal to use the highest output voltages for the high frequency at the beginning of the heating process, whereas the highest output voltages for the medium frequency should be applied towards the end.

In order to provide a documentation of the history of the inductive heating process, Figure 6.3 shows the temperature distribution in a quarter-tooth of the workpiece at various stages of the process from the beginning (top left) to the very end (bottom right). One can clearly recognize the advantages of the optimally controlled dual frequency method in so far as the desired temperature is almost uniformly achieved in the prespecified region of interest. 

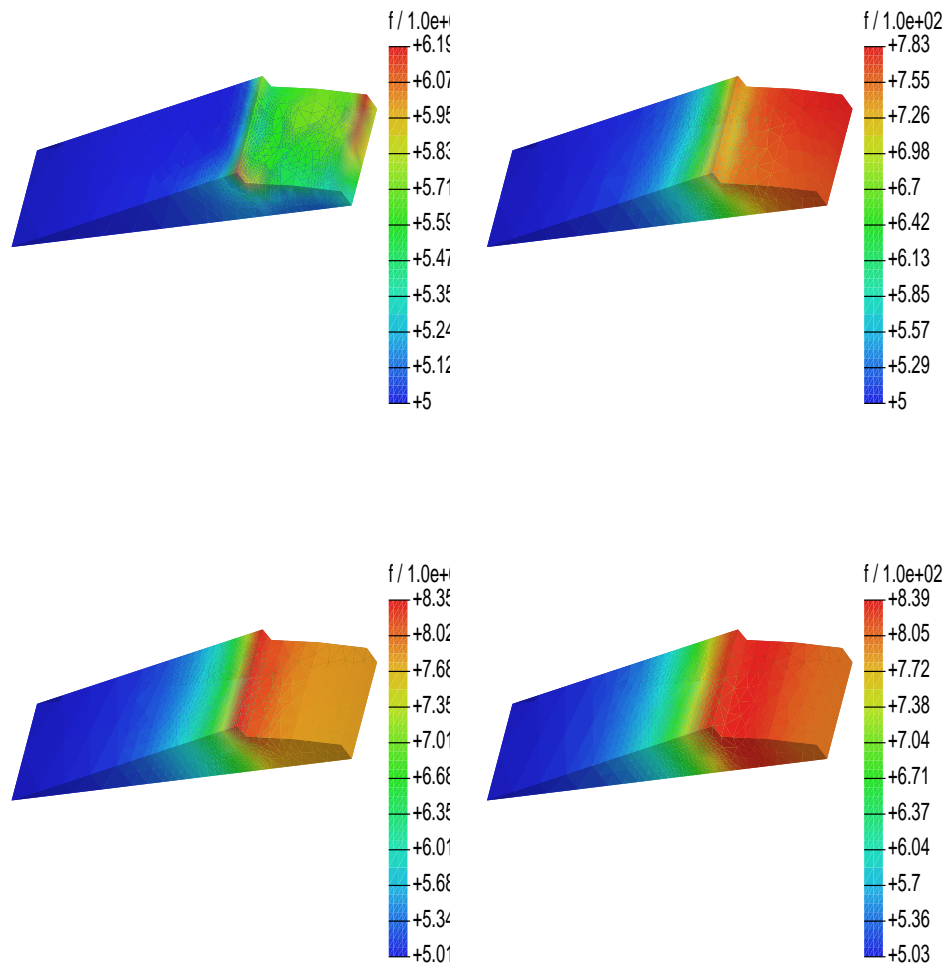

FIG. 6.3. History of the inductive heating process: Temperature distribution in a quarter-tooth at $t=0.04 \mathrm{~s}$ (top left), $t=0.80 \mathrm{~s}$ (top right), $t=1.40 \mathrm{~s}$ (bottom left), and $t=2.00 \mathrm{~s}$ (bottom right).

\section{REFERENCES}

[1] H. Ammari, A. Buffa, and J.-C. Nédélec; A justification of eddy currents model for the Maxwell equations. SIAM J. Appl. Math. 60, 1805-1823, 2000.

[2] F. Bay, V. Labbe, Y. Favennec, and J.L. Chenot; A numerical model for induction heating processes coupling electromagnetism and thermomechanics. Int. J. Numer. Meth. Engrg. 58, 839-867, 2003.

[3] P. Bristiel; Modélisation magnétothermique, métallurgique at mécanique de la trempe superfcielle après chauffage par induction appliquée aux vilebrequins. PhD Thesis, ENSAM CER de Bordeaux, 2001.

[4] E. Casas; Control of an elliptic problem with pointwise state constraints. SIAM J. Control Optim. 24, 1309-1318, 1986.

[5] C. Chaboudez, S. Clain, R. Glardon, D. Mari, J. Rappaz, and M. Swierkosz; Numerical modeling in induction heating for axisymmetric geometries. IEEE Trans. Mag. 33, 739-745, 1997.

[6] S. Clain, J. Rappaz, J. Swierkosz, and R. Touzani; Numerical modeling of induction heating for two-dimensional geometries. Math. Mod. Meth. Appl. Sci. 3, 805-822, 1993.

[7] E. J. Davies; Conduction and induction heating. Number 0-86341-174-6 in IEE Power Engineering Series 11. Peter Peregrinus, London, 1990.

[8] P.-E. Druet, O. Klein, J. Sprekels, F. Tröltzsch, and I. Yousept; Optimal control of 3D stateconstrained induction heating problems with nonlocal radiation effects. Preprint No. 1422, Weierstrass Institute for Applied Analysis and Stochastics, Berlin, 2009. 
[9] L.R. Egan and E.P. Furlani; A computer simulation of an induction heating system. IEEE Trans. Mag. 27, 4343-4354, 1991.

[10] I. Ekeland and R. Temam; Convex Analysis and Variational Problems. SIAM, Philadelphia, 1999.

[11] A.V. Fursikov; Optimal Control of Distributed Systems: Theory and Applications. American Math. Society, Providence, 1999.

[12] M. Hintermüller, K. Ito, and K. Kunisch; The primal-dual active set strategy as a semismooth Newton method. SIAM J. Optim. 13, 865-888, 2003.

[13] R.H.W. Hoppe and R. Kornhuber; Numerical simulation of induction heating processes by multi-grid techniques. In: Numerical Methods in Thermal Problems, Vol. VI (R.W.Lewis; ed.), pp. 1142-1152, Pineridge Press, Swansea, 1989.

[14] R.H.W. Hoppe and R. Kornhuber; Multi-grid solution of two coupled Stefan equations in induction heating of large steel slabs. Int. J. Numer. Meth. Eng. 30, 779-801, 1990.

[15] C.T. Kelley; Iterative Methods for Optimization. SIAM, Philadelphia, 1999.

[16] D. Labridis and P. Dokopoulos; Calculation of eddy current losses in nonlinear ferromagnetic materials. IEEE Trans. Magnetics 25, xxx-xxx, 1989.

[17] D. Landek, F. Cajner, and T. Filetin; Computer simulation of induction surface hardening axially symmetric workpieces. J. Phys. IV France 120, 499-506, 2004.

[18] I. Magnabosco, P. Ferro, A. Tiziani, and F. Bonollo; Induction heat treatment of a ISO C45 steel bar: Experimental and numerical analysis. Comput. Materials Sci. 35, 98-106, 2006.

[19] K. Matsui, H. Hata, H. Kadogawa, and K. Yoshiyuki; Research on practical application of dual frequency induction hardening to gears. JSAE Review 19, 358-360, 1998.

[20] P. Monk; Finite Element Methods for Maxwell's equations. Clarendon Press, Oxford, 2003.

[21] J.-C. Nédélec; Mixed finite element in $\mathbb{R}^{3}$. Numer. Math. 35, 315-341, 1980.

[22] B. Paya, V. Fireteanu, A. Spahiu, and C. Guérin; 3D magneto-thermal computations of electromagnetic induction phenomena. COMPEL 22, 744-755, 2003.

[23] H.-J. Peter and W. Schwenk; Applications for surface induction hardening using SDF induction heat treating. Elektrowärme International 60, 13-18, 2002.

[24] E. Rapoport and Y. Pleshivtseva; Optimal Control of Induction Heating Processes. CRC Press, Boca Raton, 2006.

[25] M. Renardy and R.C. Rogers, An Introduction to Partial Differential Equations. Springer, Berlin-Heidelberg-New York, 1993.

[26] W. Schwenk; Simultaneous dual-frequency induction hardening. Heat Treating Process, April/May 2003, 35-38, 2003.

[27] R.L. Stoll; The Analysis of Eddy Currents. Oxford University Press, Oxford, 1974.

[28] L. Tartar; Introduction to Sobolev Spaces and Interpolation Theory. Springer, BerlinHeidelberg-New York, 2007.

[29] F. Tröltzsch; Optimal Control of Partial Differential Equations. Theory, Methods, and Applications. American Mathematical Society, Providence, 2010. 